\title{
Reliability and Energy-efficiency in IEEE 802.15.4/ZigBee Sensor Networks: An Adaptive and Cross-layer Approach
}

\author{
Mario Di Francesco, Giuseppe Anastasi, Marco Conti, Sajal K. Das, and Vincenzo Neri
}

\begin{abstract}
A major concern in wireless sensor networks (WSNs) is energy conservation, since battery-powered sensor nodes are expected to operate autonomously for a long time, e.g., for months or even years. Another critical aspect of WSNs is reliability, which is highly application-dependent. In most cases it is possible to trade-off energy consumption and reliability in order to prolong the network lifetime, while satisfying the application requirements. In this paper we propose an adaptive and cross-layer framework for reliable and energy-efficient data collection in WSNs based on the IEEE 802.15.4/ZigBee standards. The framework involves an energy-aware adaptation module that captures the application's reliability requirements, and autonomously configures the MAC layer based on the network topology and the traffic conditions in order to minimize the power consumption. Specifically, we propose a low-complexity distributed algorithm, called ADaptive Access Parameters Tuning (ADAPT), that can effectively meet the application-specific reliability under a wide range of operating conditions, for both single-hop and multi-hop networking scenarios. Our solution can be integrated into WSNs based on IEEE 802.15.4/ZigBee without requiring any modification to the standards. Simulation results show that ADAPT is very energy-efficient, with near-optimal performance.
\end{abstract}

Index Terms-Sensor networks; IEEE 802.15.4; ZigBee; crosslayer; adaptive data collection; reliability; energy efficiency.

\section{INTRODUCTION}

W IRELESS sensor networks (WSNs) are being deployed in many real-life applications, such as environmental monitoring, security and surveillance, industrial automation and control [1]. This has been possible due to the advent of: (i) the IEEE 802.15.4 standard [2], which defines the physical and medium access control (MAC) layers of the protocol stack;

Manuscript received 17 October 2010; revised 18 February 2011. This work has been partially supported by NSF Grants IIS-0326505, CNS-0721951, CNS-0916221, CNS-1049652 and CNS-1050618, and by a grant from EADS North America. The work of S. K. Das is also supported by (while serving at) the National Science Foundation. Any opinion, findings, and conclusions or recommendations expressed in this material are those of the authors and do not necessarily reflect the views of the National Science Foundation.

M. Di Francesco and S. K. Das are with the Center for Research in Wireless Mobility and Networking (CReWMaN) at the Department of Computer Science and Engineering, University of Texas at Arlington, Arlington, TX 76019 USA (e-mail: \{mariodf,das\}@uta.edu).

Giuseppe Anastasi is with the Department of Information Engineering, University of Pisa, Pisa, Italy (email: g.anastasi@ing.unipi.it).

Marco Conti is with the Institute of Informatics and Telematics (IIT) at the National Research Council, Pisa, Italy (e-mail: marco.conti@iit.cnr.it).

Vincenzo Neri is with Ingegneria dei Sistemi (IDS), Avionics Laboratory, Pisa, Italy (e-mail: vinc.neri@gmail.com).

Digital Object Identifier 10.1109/JSAC.2011.1109xx. and (ii) the ZigBee specifications [3], which cover the network and application layers.

A major concern in WSNs is energy conservation [4], although reliability is also very critical [5]. Indeed, it has been shown that WSNs based on IEEE 802.15.4/ZigBee suffer from serious unreliability issues, especially when power management is enabled for conserving energy [6], [7]. Therefore, effective and efficient mechanisms should be provided to achieve reliability with a low energy expenditure. Now, different WSN applications have different reliability requirements. For instance, industrial control or military applications might require nearly $100 \%$ reliability. On the other hand, environmental monitoring applications might tolerate message loss, leading to a trade-off between energy conservation and reliability. For energy efficiency, the WSN protocol stack needs to be tuned according to the actual needs. The traffic and network conditions in a WSN are often very dynamic, due both to the noisy wireless channel and the failure probability of sensor nodes (e.g., when they run out of battery power). Thus, energy-aware and reliable data collection mechanisms should be able to adapt to the actual operating conditions [8]. In addition, they should be flexible enough to support a wide variety of operating scenarios, without any prior or global knowledge on the network topology and the traffic pattern. All these requirements make the design of energy-efficient adaptive schemes for reliable data collection a significant challenge. To this end, a cross-layer approach is definitely beneficial, since it can exploit the knowledge provided by the different layers of the protocol stack to minimize the energy expenditure [9].

In this paper we propose an adaptive and cross-layer approach for reliable and energy-efficient data collection in WSNs based on the IEEE 802.15.4/ZigBee standards. Our approach involves an energy-aware adaptation module that captures the application's reliability requirements and autonomously configures the MAC layer, based on the network topology and current traffic conditions. Specifically, we propose the ADaptive Access Parameters Tuning (ADAPT) algorithm, based on an analytical study of the IEEE 802.15.4 standard. ADAPT is simple and lightweight, and uses only information local to the sensor nodes. As a result, it is fully distributed, and has a very low complexity, thus being well suited for resource-constrained sensor nodes. Furthermore, it can be integrated into WSNs based on IEEE 802.15.4 without requiring any modification to the standard. We show that 
ADAPT is effective, in the sense that it can satisfy a target reliability constraint while consuming low energy, and its performance is near-optimal for a wide range of operating conditions, for both single-hop and multi-hop networks.

The rest of the paper is organized as follows. Section II presents the related work, and Section III introduces the IEEE 802.15.4 and ZigBee standards. Section IV evaluates the reliability and energy-efficiency of the IEEE 802.15.4 MAC protocol. Section V introduces our adaptive cross-layer framework for reliable and energy-efficient data collection, and presents ADAPT. Section VI discusses the simulation setup, followed by the performance evaluation in Section VII (single-hop scenario) and Section VIII (multi-hop scenario). Finally, Section IX concludes the paper.

\section{RELATED WORK}

There exists literature about cross-layer frameworks and adaptive approaches to data collection in WSNs. This section briefly reviews the related work.

As mentioned, energy efficiency is one of the major concerns in WSNs [4]. In addition to providing optimized solutions specific to a single layer of the protocol stack, various cross-layer approaches have been proposed in the literature [9]. Most of these approaches focus on the joint optimization of the physical and MAC layers, or the MAC and networking (e.g., routing) layers. For instance, the activity management scheme presented in [10] jointly exploits the MAC and the physical layer. In addition, only a limited number of works specifically deals with the IEEE 802.15.4/ZigBee standards. A cross-layer optimization framework is proposed in [11] based on an experimental analysis of interference in IEEE 802.15.4 networks. However, the focus is mostly on the physical layer in the form of power control. Finally, only limited literature jointly evaluates the impact of the network/application layer on the performance of IEEE 802.15.4 networks. For instance, the impact of different sleep/wakeup scheduling policies in multihop WSNs is investigated in [7], with special focus on ZigBee networks. Although the authors provide hints on how to tune the IEEE 802.15.4 MAC layer, the investigated solution is not adaptive, nor does it support application-specific reliability requirements.

Alongside, relatively less attention has been paid to reliability guarantees in WSNs. In the context IEEE 802.15.4based WSNs, many papers [12], [13], [14] highlighted that a significant share of transmitted messages may be lost due to contention, especially when the number of sensor nodes and the message size are large. In addition, [6] and [7] have shown that serious reliability issues arise when power management is enabled, even in networks with a small number of sensor nodes. It is also shown that a suitable setting of the MAC parameters can alleviate the problem, such that it is possible to achieve a target level of reliability, as well as a higher energy efficiency. However, [7], [6] are based on static (i.e., pre-defined) settings for the MAC parameters, hence they are not flexible enough to support dynamic operating conditions. In addition, a static parameter setting typically results in an energy consumption higher than necessary. An adaptive mechanism for reliable and adaptive data collection in IEEE 802.15.4-based WSNs has been proposed in [15]. This solution is based on an optimization problem built on top of an analytical model of the IEEE 802.15.4 standard [16]. As a result, the proposed approach has significant computational and storage overheads, which make it unsuitable for implementations on real sensors.

\section{A. Our contributions}

In this paper we design and develop an adaptive and crosslayer approach for data collection in IEEE 802.15.4/ZigBee WSNs. Different from most solutions available in the literature, our approach is specifically suited for the IEEE 802.15.4 and ZigBee standards. In contrast with many approaches such as [12], our scheme does not require any modification to the IEEE 802.15.4 MAC protocol, and hence can be implemented in real sensors even when the MAC protocol cannot be altered. Furthermore, unlike [7], [6], our scheme is adaptive, hence it can tailor the operating parameters - e.g., the backoff window size and the number of (re)transmissions - according to the actual traffic demands. In addition, it is flexible since it supports diverse policies for enforcing the required level of reliability. In this paper, we extend the work in [8] by considering the message loss due to a noisy channel. Furthermore, we explicitly address multi-hop scenarios, also in contrast with most of the existing solutions which are limited to single-hop (star) networks [6], [15]. Simulation results show that our proposed approach is effective, in the sense that it can satisfy a target reliability constraint while achieving low energy consumption (and low latency) in both single-hop and multi-hop scenarios, even when the message loss is high. At the best of our knowledge, this is the first solution tailored to IEEE 802.15.4/ZigBee WSNs which is able to effectively tune the MAC parameters and overcome the reliability problem of the MAC protocol [17] in an energy-efficient way.

\section{BACKGROUND: IEEE 802.15.4 AND ZIGBEE}

In the following, we will briefly introduce the IEEE 802.15.4 and the ZigBee standards, and their features relevant for the subsequent discussion in the paper.

\section{A. The IEEE 802.15.4 standard}

IEEE 802.15.4 standard [2] is for low-rate, low-power, and low-cost Personal Area Networks (PANs). The basic components of IEEE 802.15.4 networks are: the PAN coordinator (i.e., sink), which manages the entire network; one or more coordinators, which manage a cluster of nodes; and ordinary nodes that associate to some coordinator in order to participate in the network operations. Besides the simple star network, IEEE 802.15.4 also supports multi-hop topologies, such as cluster-tree and mesh.

As for the channel access, the standard defines two different functions: the beacon enabled and non-beacon enabled modes. The beacon enabled mode provides a power management mechanism based on duty-cycle, and implemented through a superframe structure bounded by beacons, i.e., special synchronization frames generated periodically by the coordinator nodes. The time between two consecutive beacons is called 
the Beacon Interval, $B I=15.36 \cdot 2^{B O}$ ms for $0 \leq B O \leq 14$, where $B O$ is the Beacon Order parameter. Each superframe consists of the Active Period and the Inactive Period. In the Active Period, nodes communicate with the coordinator they are associated with, while during the inactive period they enter a low power state to save energy. The Active Period is denoted by the Superframe Duration, $S D=15.36 \cdot 2^{S O} \mathrm{~ms}$, for $0 \leq S O \leq B O \leq 14$, where $S O$ is the Superframe Order. The SD can further be divided into the Contention Access Period (CAP) and the Collision Free Period (CFP). During the CAP, a slotted Carrier Sense Multiple Access with Collision Avoidance (CSMA/CA) algorithm is used for channel access, while in the CFP, communication occurs in a Time Division Multiple Access (TDMA) style by using a number of Guaranteed Time Slots (GTSs), pre-assigned to individual sensor nodes. In the non-beacon enabled mode, there is no superframe and no power management implemented at the MAC (although a higher layer policy can be used).

The beacon enabled mode uses a slotted CSMA/CA algorithm for channel access, i.e., all operations are aligned to backoff period slots with a duration of $320 \mu \mathrm{s}$. Upon receiving a data frame to be transmitted, the slotted CSMA/CA algorithm performs the following steps.

1) The contention window size $(C W=2)$, the number of backoff stages $(N B=0)$, and the backoff exponent (which is set to the default minimum value, i.e., $B E=$ MACMINBE) are initialized as state variables.

2) A backoff timer is initialized by using a random backoff time uniformly distributed in the range $\left[0,320 \cdot\left(2^{B E}-\right.\right.$ 1)] $\mu \mathrm{s}$.

3) The status of the wireless medium is checked through the Clear Channel Assessment (CCA).

4) If the medium is busy, the state variables are updated as follows: $N B=N B+1, B E=\min (B E+$ 1, MACMAXBE) and $C W=2$. If the number of backoff stages exceeds the maximum allowed value (i.e., $N B>$ MACMAXCSMABACKOFFs), the frame is dropped. Otherwise, the algorithm falls back to Step 2.

5) If the medium is free, then $C W=C W-1$. If $C W=0$ the frame is transmitted. Otherwise the algorithm falls back to Step 3 to perform a second CCA.

In the non-beacon enabled mode, the unslotted version of the CSMA/CA algorithm is used. Hence, operations are not aligned to the backoff period slots. In addition, the CCA operation is performed only once to check whether the channel is busy or not (i.e., $C W=1$ ).

In both cases, the CSMA/CA algorithm supports an optional retransmission scheme based on acknowledgements. When retransmissions are enabled, the destination node must send an acknowledgement just after receiving a data frame. Unacknowledged messages are retransmitted up to MACMaxFrameRetries times, and then dropped. Every time the message is (re)transmitted, the CSMA/CA algorithm starts from Step 1.

\section{B. The ZigBee specifications}

The ZigBee specifications [3] provide standard solutions for sensor and control systems, with emphasis on interoperability of different vendor platforms. The ZigBee protocol stack is grounded on the physical and MAC layers as defined by the IEEE 802.15.4 standard, and consists of the network and the application layers.

The network layer provides means for network management, security and routing. Network management deals with how to create a network and handle the addition (and removal) of devices. This is accomplished through an association (or disassociation) procedure which enables nodes to become members of a network (or rather leave it). Different network topologies are supported, such as star, cluster-tree, and mesh. For cluster-tree networks, the specifications define both a distributed addressing scheme, as well as a hierarchical routing based on the tree structure. The application layer is composed of two major elements. On the one hand, the Application Support Layer (ASL) provides device and service discovery, as well as binding mechanisms between devices and services. On the other hand, the Application Framework (AF) contains the higher layer components, namely the Application Objects (AOs), which implement the specific application as defined by the user.

As for the power management, the ZigBee specifications support the IEEE 802.15.4 beacon enabled mode for both the star and cluster-tree network topologies. In cluster-tree networks, the following constraint needs to be satisfied: the active period of different coordinators in the network cannot be overlapped. In other words, the active periods of (interfering) coordinators have to be assigned in a TDMA fashion. The beacon enabled mode is not supported for mesh networks, where a power management policy has to be defined on top of the IEEE 802.15.4 non-beacon enabled mode.

\section{Characterization of The IEEE 802.15.4 MAC}

In this section we will characterize the performance of the IEEE 802.15.4 MAC in order to derive a few guidelines for the design of adaptive strategies for reliable and energy-efficient operations. To this end, we first introduce an analytical model of the IEEE 802.15.4 MAC, and then evaluate the impact of the most significant MAC parameters on the reliability and the energy efficiency of the network.

\section{A. An analytical model of the IEEE 802.15.4 MAC}

Before presenting the model, let us first introduce the assumptions behind the subsequent analysis and evaluation. We consider a star topology where one node acts as a PAN coordinator, while the other nodes act as ordinary nodes. We assume that the nodes access the channel according to the beacon enabled mode, and use acknowledgements. We also assume that the ordinary nodes operate in saturation conditions (i.e., they always have a message to send while being active), and power off their radio ${ }^{1}$ during the idle times of channel access (including the backoff) for better efficiency, similarly to the energy model in [18], [17].

We adapted the model in [16] by taking into account the message loss due to channel errors. The derivations of the formulas presented below require several algebraic manipulations, therefore we do not report them here for lack of

\footnotetext{
${ }^{1}$ Clearly, the coordinator has to be always active in order to receive data.
} 
space. They can be derived by extending those in [16] with the parameter $p_{\text {loss }}$ which represents the probability that a message is discarded due to channel errors. In detail, we consider the delivery ratio $R$ as a measure of reliability as follows:

$$
R=1-p_{c a f}-p_{r t x},
$$

where $p_{c a f}$ is the probability that a message is discarded due to a channel access failure (i.e., exceeded number of backoffs), and $p_{r t x}$ is the probability that a message is discarded due to exceeding the number of retransmissions. Specifically,

$$
p_{\text {caf }}=\frac{x^{m+1} \cdot\left(1-\tilde{y}^{n+1}\right)}{1-\tilde{y}}
$$

where: $x$ is the probability that the channel is busy (either at the first or at the second CCA); $\tilde{y}$ is the probability that a message is not correctly received at the destination (i.e., due to either collisions or channel errors); $m \triangleq$ MACMAXCSMABACKOFFS; and $n \triangleq$ MACMAXFRAMERETRIES. In addition, $p_{r t x}=\tilde{y}^{n+1}$ with $\tilde{y}=\left(p_{c}+p_{\text {loss }}\right) \cdot\left(1-x^{m+1}\right)$, where $p_{c}$ is the probability of collision (i.e., that more than one node transmit during the same slot) and $p_{\text {loss }}$ is the probability of message loss due to the noisy channel.

We used the following equation to characterize the power consumption:

$$
\begin{aligned}
P= & \frac{P_{s} \tau}{2}\left(\frac{(1-x)\left(1-(2 x)^{m+1}\right)}{(1-2 x)\left(1-x^{m+1}\right)} W_{0}-1\right) \\
& +P_{c s}(2-\alpha) \tau+(1-\alpha)(1-\beta) \tau \\
& \cdot\left(P_{t} L+P_{s}+L_{a c k}\left(P_{r}\left(1-p_{c}\right)\left(1-p_{\text {loss }}\right)\right.\right. \\
& \left.+P_{s}\left(1-\left(1-p_{c}\right)\left(1-p_{\text {loss }}\right)\right)\right)
\end{aligned}
$$

where: $P_{r}, P_{t}, P_{c s}$ and $P_{s}$ respectively denote the power consumption for the receive, transmit, channel sensing, and sleep radio states; $W_{0} \triangleq 2^{\mathrm{MACM} \text { INBE }}$ is the initial backoff window size; $L$ and $L_{a c k}$ are, respectively, the message and the acknowledgement durations; finally, the remaining parameters $\alpha, \beta$, and $\tau$ are the channel access probabilities as defined in [16].

Observe that Equation (2) is composed of three terms: the first characterizes the power consumption due to the backoff procedure; the second accounts for the power required by the carrier sensing; finally, the last term embeds the power needed for the data exchange, which includes both the transmission of the message and the reception of the corresponding acknowledgement.

The model described by Equations (1) and (2) is completely specified once the technology-dependent parameters (e.g., the power consumption of the radio in the different states) and the traffic-dependent parameters (e.g., number of nodes, message size, and message loss probability) are all set. Specifically, the model consists of a system of non-linear equations where the unknowns are represented by the access parameters $\alpha, \beta$, and $\tau$.

The complexity of the analytical model forced us to evaluate it numerically. The numerical study of the model provided us very interesting information about the impact of the MAC parameters on the system performance (cfr. to the next section). Even though we performed such numerical evaluation for a wide range of operating conditions (in terms of number of

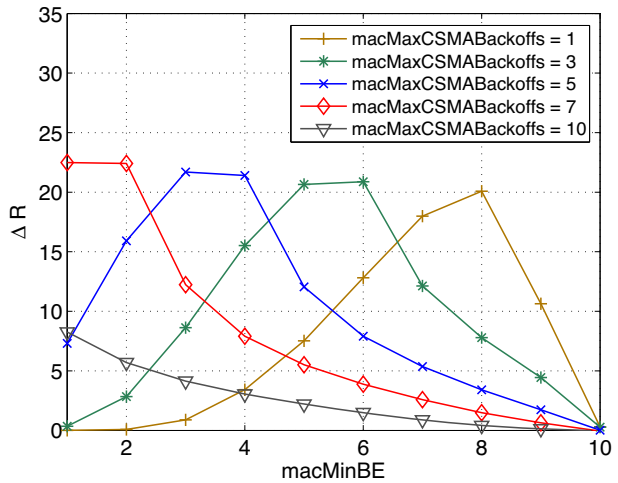

(a)

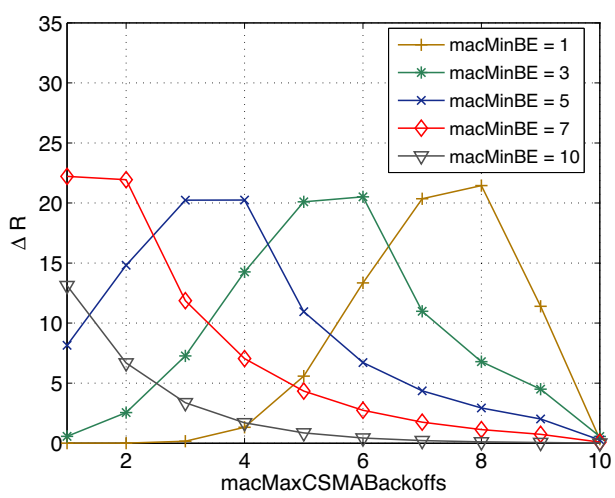

(b)

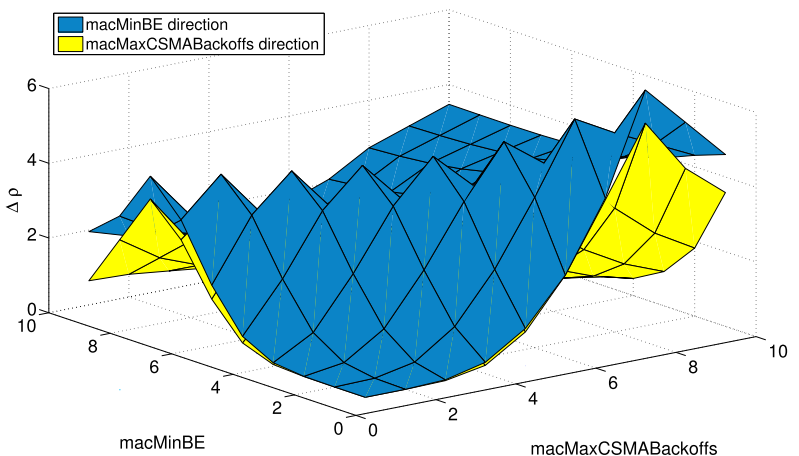

(c)

Fig. 1. Discrete gradient of the reliability with respect to: (a) MACMINBE and (b) MACMAXCSMABACKOFFS; (c) discrete gradient of the efficiency function $\rho$. The plots have been obtained by considering a network topology made of 40 nodes (in addition to the sink), a MAC payload size of 100 bytes, and a $0 \%$ message loss.

nodes and message loss rate), for clarity in the next discussion we present detailed results only for a network of 40 nodes (in addition to the sink), where the messages have a MAC payload size of 100 bytes and are never discarded due to channel errors. The chosen reference scenario represents a loaded network, where reliability issues start being critical.

\section{B. Impact of MAC parameters}

In the following, we characterize the impact of individual MAC parameters on the delivery ratio and power consumption. 
We will restrict our analysis to MACMINBE (the minimum backoff exponent), MACMAXCSMABACKOFFS (the maximum number of backoffs, i.e., transmission attempts), and MACMAXFRAMERetries (the maximum number of retransmissions), since they have the most significant impact on the considered metrics [6]. We will also assume that MACMAXBE is large enough so that its effect on the performance is not significant. In fact, the maximum backoff exponent would affect the CSMA/CA algorithm only after a large number of transmission attempts, and only when the minimum backoff exponent is large (cfr. also Section III-A).

For a loaded network (i.e., under the conditions mentioned in the previous section), the following observations hold.

\section{Observation 1 (Monotonic Reliability)}

The reliability $R$ increases monotonically as MACMINBE, MACMAXCSMABACKOFFS, and MACMAXFRAMERETRIES increase in their values.

Observation 1 is rather intuitive. In fact, an increase in MACMINBE results in a lower probability that the channel is found busy, since the transmission attempts by different nodes are spread over a larger time (driven by the backoff window size). In addition, increasing MACMAXCSMABACKOFFS increases the number of transmission attempts, thus enhancing the impact of the backoff exponent as well (cfr. Section III-A). Similarly, increasing MACMAXFrameREtries also increases the chance of successful transmissions, since the basic transmission mechanism is repeated whenever a collision occurs or a message is dropped due to channel errors.

A simple corollary of Observation 1 is that the highest reliability can be obtained by using the highest values of the MAC parameters. However, using the maximum values might be not very meaningful, since the trend of $R$ may not be the same for the different MAC parameters. To this end, we analyzed the finite difference $\Delta \mathrm{R}(\widetilde{m})=\mathrm{R}(\widetilde{m}+1)-\mathrm{R}(\widetilde{m})$, where $\widetilde{m}$ is one of the MAC parameters under consideration. The resulting insights are summarized as follows.

\section{Observation 2 (Bounded MAC Parameters)}

The reliability $R$ does not increase significantly for values of MACMINBE, MACMAXCSMABACKOFFS and MACMAXFRAMERETRIES above certain thresholds.

We have verified, by evaluating $R$ for the different MAC parameters, that there is no advantage in using values of MACMINBE, MACMAXCSMABACKOFFS and MACMAXFrameRetries beyond certain thresholds. These thresholds depend on the actual operating conditions, which include the number of nodes, the message size, and the message loss rate. However, there are some bounds on the values which provide a meaningful increase in the reliability. As a reference, we show the trend of $\Delta \mathrm{R}(\widetilde{m})$ for $\widetilde{m} \in\{$ MACMINBE, MACMAXCSMABACKOFFS $\}$, when retransmissions are not enabled, as shown in Figures 1a and 1b, respectively. We observe that values of the MAC parameters above 10 make $\Delta \mathrm{R}(\widetilde{m}) \approx 0$. Since the reliability is monotonic (Observation 1), the maximum value of $R$ is reached at the values corresponding to the thresholds. Finally, we evaluated numerically MACMAXFRAMERETRIES for a wide range of operating conditions, with special reference to the message loss rate. The evaluation showed that it does not affect the reliability when the message loss is low (e.g., below 10\%). In addition, values of MACMAXFRAMERETRIES higher than 3 do not provide significant benefits, even when the message loss is high (e.g., 30\%).

Although Observations 1 and 2 provide important clues on how the MAC parameters impact on reliability, they actually do not consider the related energy consumption. Recall that our design goal is to trade off reliability for energy efficiency, while satisfying the application requirements, whenever possible. Therefore, we define the efficiency $\rho(\widetilde{m})=R(\widetilde{m}) / P(\widetilde{m})$ as a tradeoff metric which jointly considers the reliability and power consumption, and study its discrete gradient $\Delta \rho(\widetilde{m})$. Note that the parameter $\rho$ is crucial in evaluating the energyreliability tradeoff, as well as the overall efficiency of a given set of MAC parameters. The related findings are summarized by the following observation.

\section{Observation 3 (MAC Parameters Efficiency)}

MACMINBE provides a higher efficiency than MACMAXCSMABACKOFFS, in the sense that it can increase the reliability with a lower power consumption.

The gradient $\mathrm{R}(\widetilde{m})$, shown in Figure 1c, illustrates the different impact of the MACMINBE and MACMAXCSMABACKOFFS on energy efficiency. In fact, even though both MAC parameters increase the reliability, MACMAXCSMABACKOFFS results in a higher energy consumption (i.e., in a lower efficiency) than MACMINBE. In fact, nodes have to perform a clear channel assessment (CCA) at the end of each backoff stage, and checking the channel for activity incurs significant energy consumption. On the other hand, increasing the backoff window size results in a longer time during which the transceiver is sleeping (remember that the transceiver is put in sleep mode during idle times in our approach).

As for retransmissions, a similar study showed that MACMAXFrameRetries has a major contribution to the power consumption, rather than to the reliability, as compared to the other MAC parameters. This happens because a higher number of retransmissions results in repeating the single channel access multiple times. In addition, significant energy is consumed for transmitting a message whenever a collision or a channel error occurs. Finally, when the maximum number of retransmissions exceeds 3, its impact on the efficiency $\rho$ is almost negligible for a variety of operating conditions, even when the message loss is high (e.g., 30\%), thus substantially corroborating Observation 2.

\section{ADaptive Access Parameters Tuning (ADAPT)}

Our solution to adaptive data collection is cross-layer, and involves an adaptation module which interacts with the different layers of the WSN protocol stack (see Figure 2). Our scheme follows the approach in [19], where a vertical component is added to the layered architecture to facilitate the sharing of information between different layers of the protocol stack. This avoids duplicating efforts to collect internal state information, and leads to a more efficient system design, as the information collected by the adaptation module can be used for optimizing the protocols' functions [20]. As a result, the information coming from one layer can be exploited for 


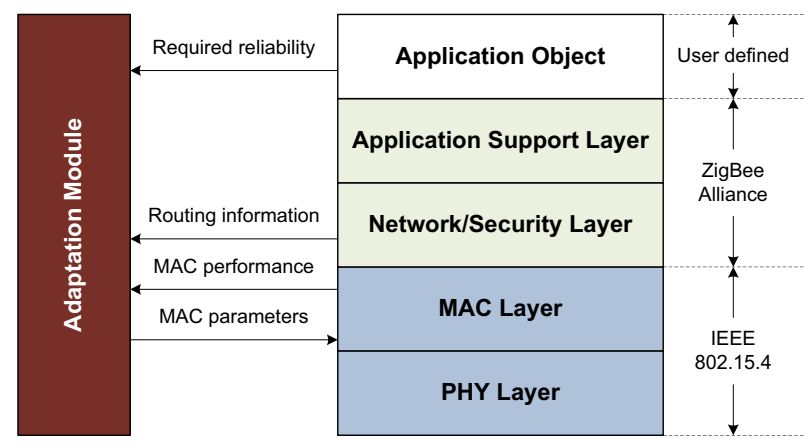

Fig. 2. The cross-layer adaptation module.

tuning the operations of protocols residing in a different (nonadjacent) layer. Specifically, the adaptation module obtains a target level of reliability (in terms of the required delivery ratio) from the application. In order to satisfy the required reliability, the adaptation module continuously monitors the performance of the MAC layer, and provides feedback on the current operating conditions by properly tuning the MAC parameters. In addition, in multi-hop networks, it exploits the information about the network topology, made available by the routing layer, for mapping the end-to-end reliability constraints to the link-level parameters (refer to section VIII for the details).

In the following, we present an adaptive scheme for reliable and energy-efficient data collection in WSNs. Our goal is to satisfy the reliability requirement specified by the application with the help of a distributed and low-overhead algorithm that consumes low energy, with specific reference to the IEEE 802.15.4 and ZigBee standards [2], [3]. To this end, we propose a heuristic algorithm called ADaptive Access Parameters Tuning (ADAPT), which estimates the current traffic conditions, and changes MAC parameters according to the required level of reliability. In the next sections, we will first introduce how reliability can be estimated, and then present detailed monitoring and control schemes to address message losses due to various factors.

\section{A. Estimation of reliability}

Before proceeding further, let us first clarify the two major factors affecting the reliability of communication in the considered scenario, namely contention (or congestion) and channel errors. Contention occurs whenever more than one node attempt to access the channel simultaneously, and becomes more serious when the amount of messages to be transmitted is significant. Severe contention leads to message loss due to either collisions or failure in the CSMA/CA algorithm, implying that the channel is always found busy in a given time frame, hence that the maximum number of backoff stages is exceeded. On the other side, channel errors affect actually transmitted messages, and are independent of contention. Since contention and channel errors are distinct factors, reliability monitoring and control schemes should address them according to their specific characteristics.

Measuring the impact of these two factors is indeed challenging, since it generally requires the knowledge of the network topology and the traffic pattern. However, gathering

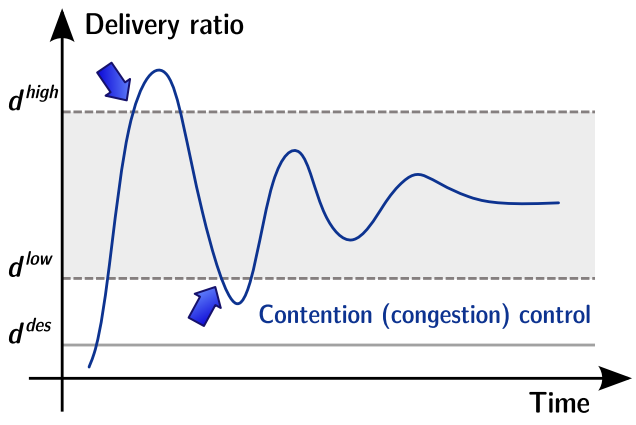

(a)

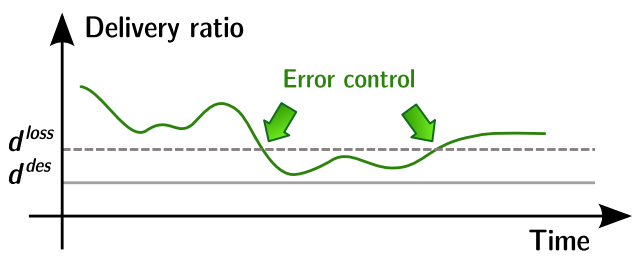

(b)

Fig. 3. Estimation-based adaptation of the delivery ratio: (a) congestion control and (b) error control.

such global information incurs excessive overhead, in terms of both energy and bandwidth consumption, and hence is not usually suitable to WSN scenarios where resources and operating conditions can quickly and dynamically change with time [17], [4]. A better approach would rather be to exploit local measurements, so that the reliability control can be effectively performed in a distributed fashion. Unfortunately, local measurements provide only a limited characterization of the operating conditions for the estimation of contention and congestion.

Individual sensor nodes can measure their own experienced reliability, in terms of the message delivery ratio, defined as the number of messages acknowledged by the destination to the number of sent messages. Such delivery ratio jointly characterizes the losses due to both contention and channel errors. However, each node can evaluate more specifically if a message has been dropped because the maximum number of backoff stages or the maximum number of retransmissions has been exceeded. In the first case, the channel is sensed busy at every transmission attempt, i.e., during every CCA after the expiration of the backoff timer. As a result, the message is discarded at the MAC layer of the sender without actually being transmitted over the channel. This kind of message loss is clearly related to contention, as it is only triggered by the channel being busy (hence used by other nodes) for long time.

In the second case, either the message collided with transmissions by other nodes at the destination or it was corrupted owing to channel errors. Since the IEEE 802.15.4 MAC does not provide an RTS/CTS mechanism, collisions can happen due either to the hidden node problem [21] or actual simultaneous transmissions (i.e., nodes which pick the very same backoff period slot after sensing the channel clear). If we assume that the carrier sensing range is at least twice than the transmission range, as shown by real experiments [22], collisions can only result from simultaneous transmissions. However, the probability of such event is very low, especially when the number of transmission attempts, the backoff win- 


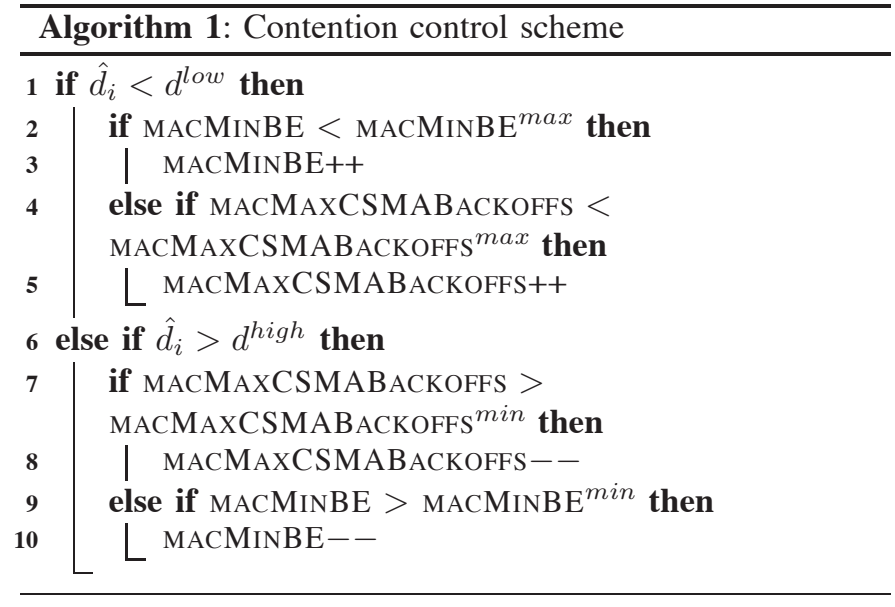

dow size, and the maximum number of transmission attempts are high. As a result, collisions due to hidden nodes can be neglected, and the message loss generated by an excessive number of retransmissions can be considered due only to channel errors.

In the following, we will exploit such considerations to decouple the impact of contention and channel errors on the reliability. Specifically, we will present two control schemes which are based on local measurements and consist of tuning strategies grounded on the analytical results described in Section IV-B, with special reference to the tradeoff between reliability and energy consumption. Each of these control schemes is performed during every Beacon Interval (BI) by the individual sensor nodes, and is applied independently from the other.

\section{B. Contention control}

The contention control scheme starts from a measured (actual) delivery ratio, $d_{i}$, corresponding to the $i$-th BI, obtained as the ratio between the number of messages acknowledged by the destination and the number of messages sent by the source node. In order to make ADAPT less sensitive to sudden variations in the measured delivery ratio, a smoothing technique is used to obtain the estimated delivery ratio, $\hat{d}_{i}$. More specifically, an exponential moving average is applied as follows:

$$
\hat{d}_{i}=\delta \cdot \hat{d}_{i-1}+(1-\delta) \cdot d_{i}
$$

where $\delta \in[0,1]$ is a memory factor.

In addition, on the basis of the level of reliability $d^{\text {des }}$ required by the application, ADAPT uses two thresholds, $d^{l o w}$ and $d^{\text {high }}$. The motivation behind the definition of these thresholds is as follows. At any time, the delivery ratio should be at least equal to $d^{d e s}$. To better react to the decrease in the delivery ratio, we define a lower threshold $d^{\text {low }}>d^{\text {des }}$ so that the adaptation can be triggered before the actual delivery ratio drops below $d^{d e s}$. On the other hand, we define a higher threshold $d^{\text {high }}$ to avoid excessive energy expenditure, given the desired level of reliability. The main goal is to keep the actual delivery ratio over $d^{d e s}$ and within the reliability region defined by the two thresholds whenever possible, as illustrated in Figure 3a. In addition, ADAPT should quickly react to variations in the operating conditions due to transient phases. In order to have a more flexible solution, we define the two thresholds as follows:

$$
\begin{aligned}
d^{\text {low }} & =d^{\text {des }} \cdot(1+\sigma) \\
d^{\text {high }} & =d^{\text {des }} \cdot(1+\sigma+\gamma)
\end{aligned}
$$

based on the parameters $\sigma \in\left(0, \frac{1}{d^{d e s}}-1\right)$ and $\gamma \in$ $\left(0, \frac{1}{d^{d e s}}-1-\sigma\right)$, respectively, representing the (weighted) distance from $d^{\text {des }}$ and the width of the reliability region.

On the basis of the estimated delivery ratio, a MAC tuning strategy is applied to control contention. Since contention is strictly related to the backoff procedure of the CSMA/CA algorithm, we only rely on the MAC parameters MACMINBE and MACMAXCSMABACKOFFS, while keeping MACMAXBE to a fixed value MACMAXBE ${ }^{d e f}$. Additional thresholds - in terms of the corresponding minimum and maximum allowed values - are also defined in order to limit the range of values achievable for both MACMINBE and MACMAXCSMABACKOFFs, in line with Observation 2. As for the actual setting of the individual parameters, ADAPT exploits the insights in Observation 3 (i.e., related to the energy-reliability tradeoff) by giving a higher priority to modifications of MACMAXBE over MACMAXCSMABACKOFFS, according to their joint impact on both reliability and energy consumption. In detail, the strategy for improving the delivery ratio is to increase MACMINBE until MACMINBE ${ }^{\max }$ is reached. Only after this point, MACMAXCSMABACKOFFS is increased up to MACMAXCSMABACKOFFS ${ }^{\max }$. The strategy for decreasing the delivery ratio is similar. First MACMAXCSMABACKOFFS is decreased until MACMAXCSMABACKOFFS ${ }^{\text {min }}$. Then MACMINBE is decreased until it reaches MACMINBE ${ }^{\text {min }}$. The contention control scheme is described in Algorithm 1.

\section{Error control}

There are basically two approaches for error control, namely adding redundancy to transmitted messages or exploiting timeouts/acknowledgements and retransmissions [21]. In general, either of these approaches can be applied, or they can even be combined in hybrid schemes. In the specific context of WSNs based on the IEEE 802.15.4 standard, the MAC layer provides error control in the form of Automatic Repeat reQuest (ARQ). Approaches based on redundancy are not applicable, since they would require modifications to the physical or MAC layer of IEEE 802.15.4, thus making the corresponding solutions not compliant with the standard. Therefore, in the following we utilize the ARQ scheme offered by the IEEE 802.15.4 MAC for error control purposes.

In order to decouple the effects of the noisy channel from other factors, we also estimate the message loss in addition to the delivery ratio. Specifically, we measure the message loss $l_{i}$, corresponding to the $i$-th $\mathrm{BI}$, as the ratio between the messages discarded due to exceeded number of retransmissions and the total number of messages sent in the last BI. Similarly to the approach used for the delivery ratio, an exponential moving average is also applied here to derive the estimated message loss $\hat{l}_{i}$ as follows:

$$
\hat{l}_{i}=\psi \cdot \hat{l}_{i-1}+(1-\psi) \cdot l_{i}
$$




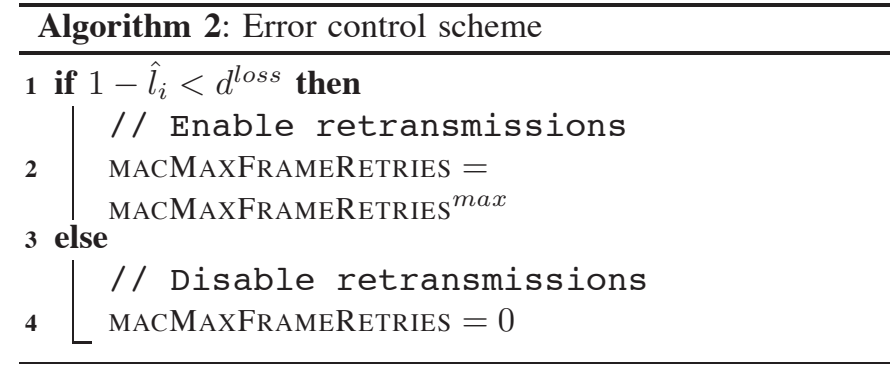

where $\psi \in[0,1]$ is a memory factor. In addition, we derive an additional threshold

$$
d^{\text {loss }}=d^{\text {des }} \cdot(1+v)
$$

where $v \in\left(0, \frac{1}{d^{d e s}}-1\right)$ is a value that indicates the sensitivity to the message loss. In this context, $d^{\text {loss }}$ represents the value of the delivery ratio which triggers the error control mechanism, similarly to how $d^{\text {low }}$ and $d^{\text {high }}$ are used for congestion control, as shown in Figure $3 \mathrm{~b}$.

The basic idea behind the actual control scheme is driven by Observations 2 and 3 as follows. Contention control alone is enough to obtain a $100 \%$ delivery ratio when the message loss is negligible [17], [8]. However, when the drop in the delivery ratio only due to message loss (i.e., the term $1-\hat{l}_{i}$ ) makes the actual delivery ratio close to the desired value $d^{\text {des }}<d^{\text {loss }}$, then retransmissions are enabled by setting MACMAXFrAMERETRIES = MACMAXFRAMERETRIES ${ }^{\max }$, otherwise they are disabled ${ }^{2}$ by setting MACMAXFRAMERETRIES $=0$. The error control scheme is described in Algorithm 2.

\section{Simulation SetuP}

For our experimental study and performance evaluation of the proposed scheme ADAPT, we used the ns2 simulator [23]. In all experiments we assumed that the IEEE 802.15.4 MAC protocol is operating on top of the $2.4 \mathrm{GHz}$ physical layer with a maximum bit rate of $250 \mathrm{Kbps}$. The transmission range was set to $15 \mathrm{~m}$, while the carrier sensing range was set to $30 \mathrm{~m}$, according to the settings in [6]. Unless otherwise specified, we assume that there is no message loss due to channel errors, although we have also conducted experiments with (correlated) message errors/losses to broaden our analysis. To this end, we considered a Gilbert-Elliot model and used an approach similar to [6]. Specifically, we used a base value of the message loss rate of $10 \%$, and the average error-burst and error-free burst sizes (i.e., average sojourn times in the bad and good states of the Gilbert-Elliot model) of 5.7 and $46.2 \mathrm{~ms}$, respectively [24]. Other values of the message loss rate were obtained by changing the average sojourn time in the bad state accordingly, while leaving all the other parameters unchanged.

We used the IEEE 802.15.4 beacon enabled mode; the beacon interval was set to $B I=125.8$ s (i.e., $B O=13$ ), and the active period was set to $S D=3.93$ s (i.e., $S O=8$ ). We assumed that the nodes send their messages just after

\footnotetext{
${ }^{2}$ Note that disabling retransmissions does not disable the acknowledgement mechanism, which is needed by the monitoring component to obtain the estimate of both the delivery ratio and the message loss.
}

TABLE I

PARAMETERS USED FOR SIMULATION

\begin{tabular}{|c|c|}
\hline Parameter & Value \\
\hline target delivery ratio $\left(d^{\text {des }}\right)$ & $80 \%$ \\
\hline memory of the delivery ratio estimator $(\delta)$ & $60 \%$ \\
\hline weighted distance of $d^{l o w}(\sigma)$ & $3 \%$ \\
\hline additional weighted distance of $d^{\text {high }}(\gamma)$ & $3 \%$ \\
\hline memory of the message loss estimator $(\psi)$ & $80 \%$ \\
\hline sensitivity to message losses $(v)$ & $2.5 \%$ \\
\hline MACMAXBE $^{d e f}$ & 10 \\
\hline MACMINBE $^{m \imath n}$ & 1 \\
\hline MACMINBE $\max ^{\max }$ & 7 \\
\hline MACMAXCSMABACKOFFS $^{m i n}$ & 1 \\
\hline MACMAXCSMABACKOFFS ${ }^{\max }$ & 10 \\
\hline MACMAXFRAMERETRIES $^{\max }$ & 3 \\
\hline
\end{tabular}

receiving a beacon from the coordinator, and used the energy model $^{3}$ in [18], [17], which is based on the Chipcon CC2420 radio [25]. We set the parameters $\sigma, \gamma$, and $v$ to suitable values obtained through a preliminary simulation study, and considered a target delivery ratio of $80 \%$. The simulation parameters are summarized in Table I.

Our analysis considered the following performance metrics.

- Delivery ratio: the ratio between the number of messages correctly received by the sink to the number of messages generated by all sensor nodes.

- Average energy per message: the average energy consumed by a single node in a Beacon Interval (BI) per single message successfully delivered to the sink.

- Average latency: the average time elapsed between the instant at which a message is transmitted at the source node, and the instant at which the same message is correctly received by the sink.

To evaluate the performance of the ADAPT algorithm, we considered three additional MAC parameter setting schemes for comparison purposes.

- Default Parameters Set (DPS): the default values specified by the IEEE 802.15.4 standard.

- Constant Parameters Set (CPS): constant values beyond the maximum ones allowed by the IEEE 802.15.4 standard $^{4}$.

- Optimal Parameters Set (OPS): values obtained from the analytical model (i.e., our modified version of that in [16] as presented in Section IV-A) by minimizing the energy consumption, under the constraint of bounded latency, below $500 \mathrm{~ms}$.

DPS is intended to show the performance of IEEE 802.15.4 without any tuning of MAC parameters, while CPS is designed to provide high reliability in most operating conditions. In contrast with DPS and CPS, which statically define the MAC parameter values, OPS is evaluated according to the actual operating conditions. Under a complete knowledge of the network parameters (such as the number of nodes or the message size) OPS represents the optimal solution, since it

\footnotetext{
${ }^{3}$ As a consequence, as also mentioned at the beginning of Section IV-A, we put the transceiver in sleep mode during idle times.

${ }^{4}$ In detail, CPS consists of the following values of the MAC parameters: MACMinBE=8, MACMAXBE=10, MACMAXCSMABACKOFFS=10, MACMAXFRAMERETRIES $=7$.
} 


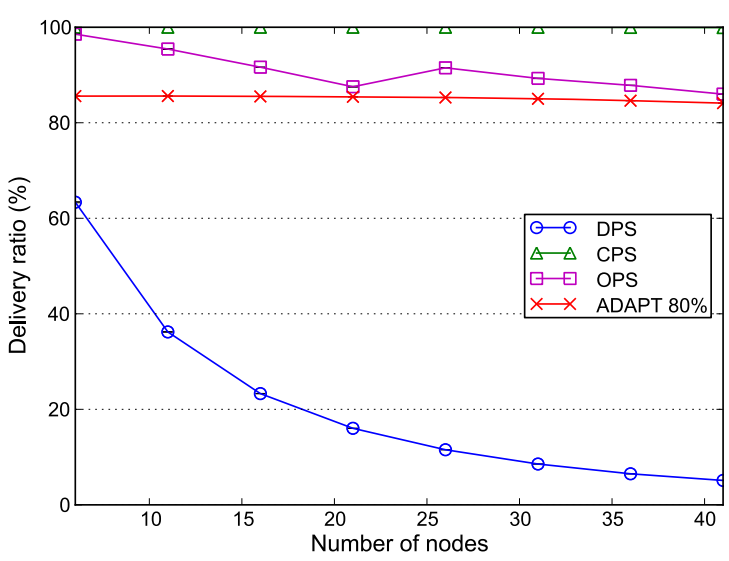

(a)

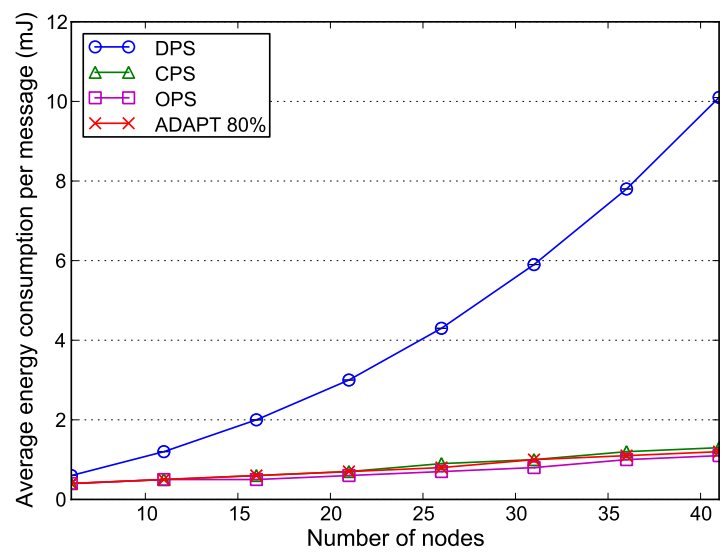

(b)

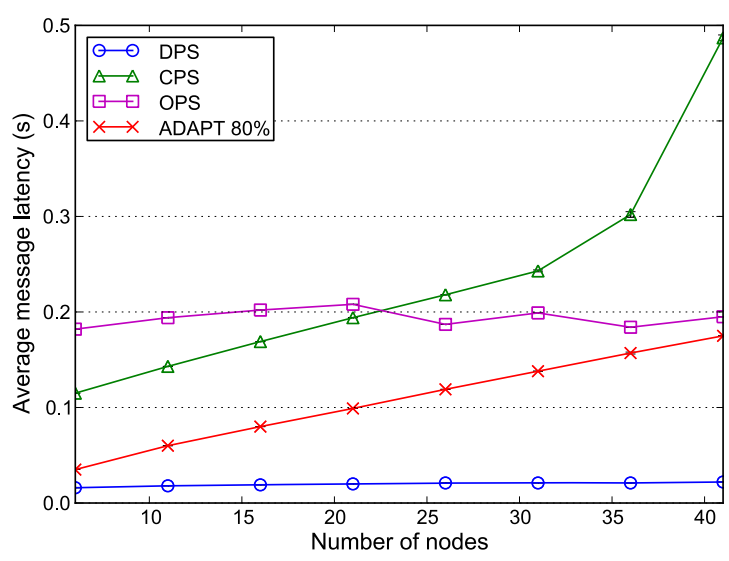

(c)

Fig. 4. Performance as a function of the number of nodes in the single-hop scenario: (a) delivery ratio, (b) energy consumption, and (c) latency.

minimizes the energy expenditure. However, the related approach is unfeasible in practice, since the operating conditions may not only be unknown, but also change with time.

In the following two sections, we will investigate the performance of the different schemes for single-hop and multi-hop networks, respectively. In all cases, we used the independent replication method and performed several simulation runs, each lasting for 1000 Beacon Intervals (BIs). The figures show the average values of the different metrics, as well as the related standard deviations (where appreciable).

\section{SINGLE-HOP SCENARIO}

As a basic single-hop topology, we considered a star network where 20 sensor nodes were placed in a circle of radius equal to $10 \mathrm{~m}$, centered at the sink node. Due to the considered radio model, all nodes are in the carrier sensing range of each other, thus collisions due to the hidden node problem are excluded. The network is composed by the sink, i.e., the PAN (Personal Area Network) coordinator, and the rest of the devices operating as ordinary nodes. All messages are sent by the ordinary nodes to the sink. Unless otherwise specified, all nodes send to the sink 10 messages per BI, so that the actual network conditions are close to saturation. In the following sections, we will analyze the performance of the different schemes first under stationary conditions, and then under dynamic conditions. We will finally conclude by characterizing the convergence of ADAPT to the target delivery ratio, as well as the impact of thresholds.

\section{A. Analysis in static conditions}

In the first set of experiments, we evaluated the impact of the number of nodes on the performance (see Figure 4). From Figure $4 \mathrm{a}$ we conclude that the performance corresponding to the default MAC parameters of IEEE 802.15 .4 (i.e., DPS) is completely unsatisfactory, because they lead to a delivery ratio even below $40 \%$ when there are more than 10 nodes, while all other schemes satisfy the required delivery ratio. Both ADAPT and CPS obtain a given reliability independent on the number of nodes, while the delivery ratio of OPS initially decreases, then increases when the number of nodes is greater than 25, and finally it decreases again. This happens because OPS selects two different sets of parameters for the two regions, depending on whether the number of nodes is below or above 25 . Figure $4 \mathrm{~b}$ shows that the energy consumption of ADAPT is almost the same as the optimal solution (i.e., OPS). Regarding the latency, as shown in Figure 4c, ADAPT has always the lowest latency among the schemes which can satisfy the target reliability. For example the latency in ADAPT is much lower than CPS, and even below that of OPS.

Let us now consider the performance as a function of the number of messages per BI (see Figure 5). As for the delivery ratio, shown in Figure 5a, the results are as follows. ADAPT has almost the same delivery ratio as OPS when the number of messages per BI is higher than 5. CPS keeps a constant delivery ratio close to $100 \%$, while DPS is always below $30 \%$. The trend of the energy efficiency, depicted in Figure $5 b$, is a bit counter-intuitive at first, since the energy consumption decreases with the number of messages per BI for all considered schemes. Actually this behavior can be explained as follows. As the number of messages increases, the total time spent by the sensor nodes for accessing the channel increases as well. As a result, contentions and collisions are reduced, and nodes spend more time in the idle mode, which results in a lower energy expenditure. As for the latency, 


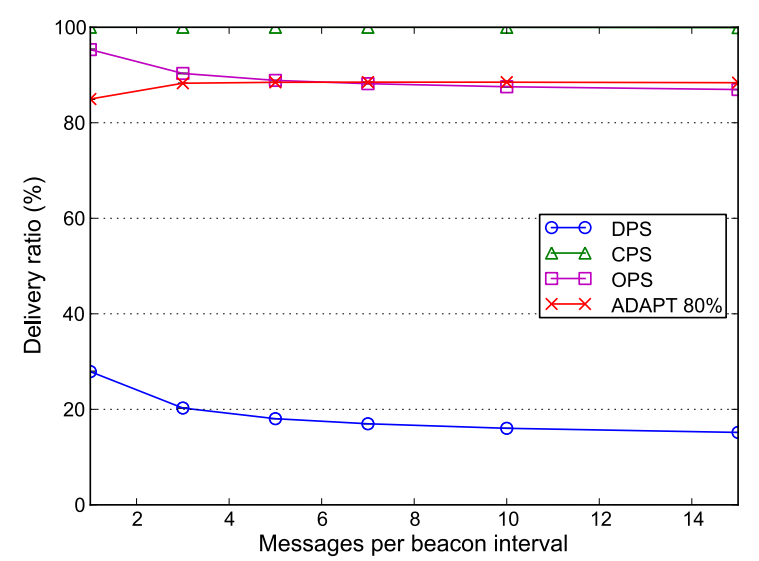

(a)

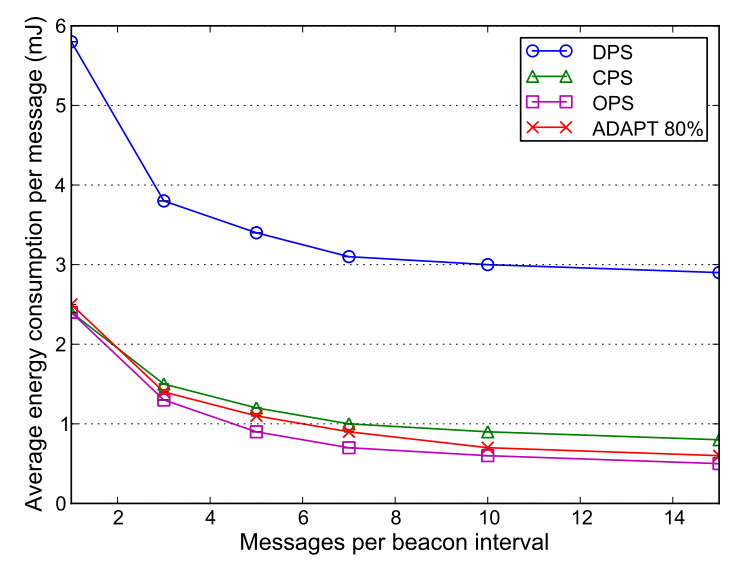

(b)

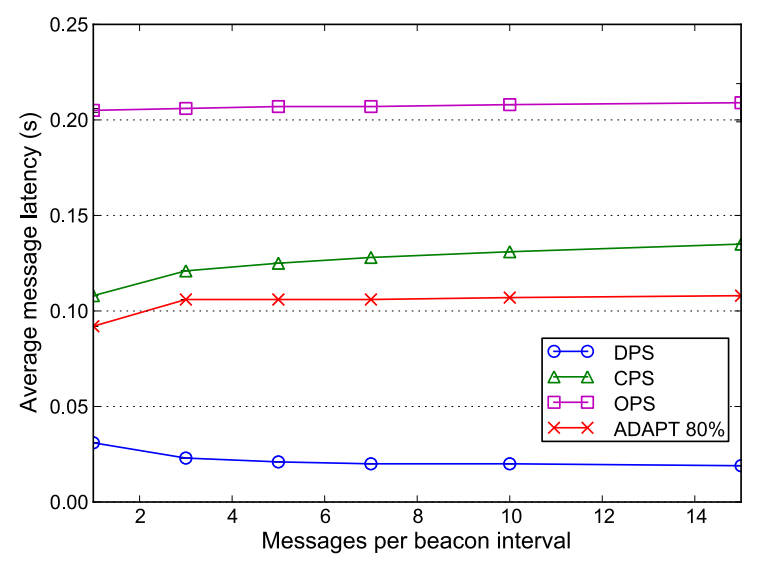

(c)

Fig. 5. Performance as a function of the number of messages per BI in the single-hop scenario: (a) delivery ratio, (b) energy consumption, and (c) latency.

shown in Figure 5c, all schemes are less sensitive to the increase in the number of messages, and ADAPT exhibits the lowest latency (less than $110 \mathrm{~ms}$ ) among all schemes satisfying the target level of reliability.

Finally, the performance as a function of the message loss rate is shown in Figure 6. The results substantially confirm

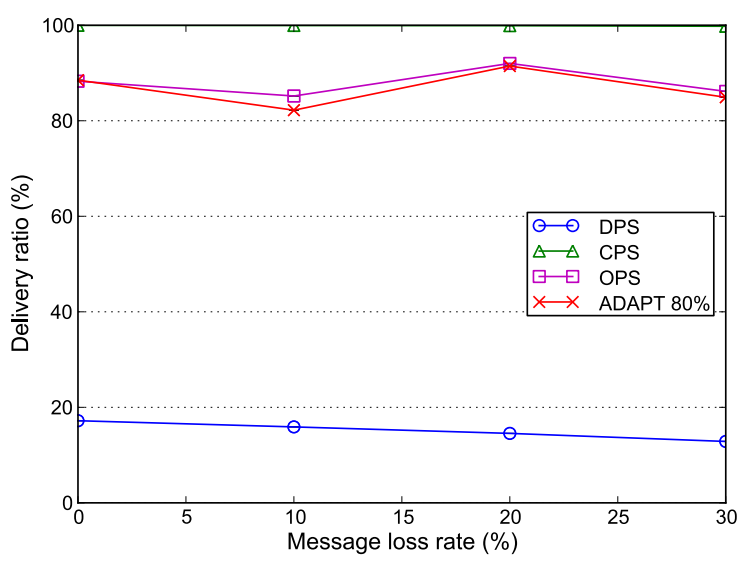

(a)

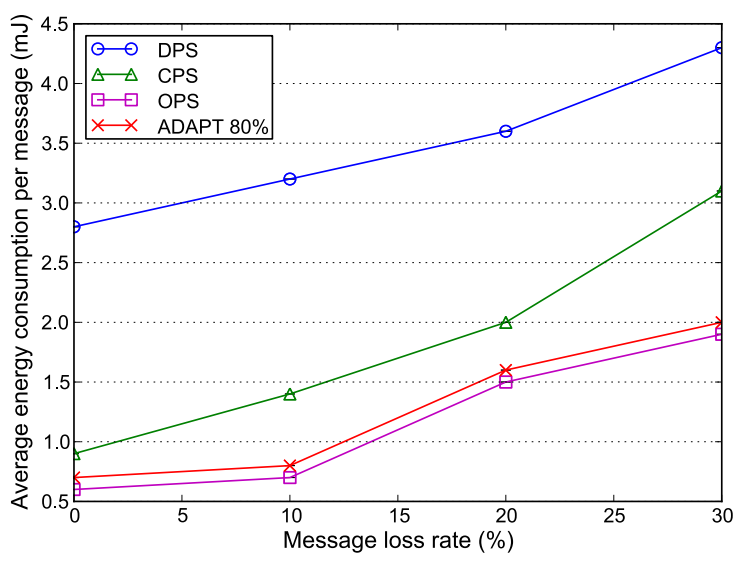

(b)

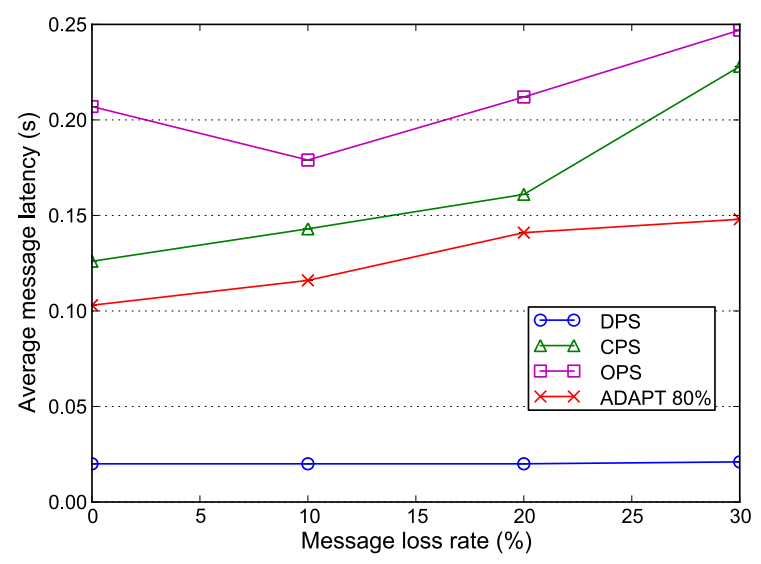

(c)

Fig. 6. Performance as a function of the message loss rate in the single-hop scenario: (a) delivery ratio, (b) energy consumption, and (c) latency.

the previous findings. Figure $6 \mathrm{a}$ demonstrates that for both OPS and ADAPT, the delivery ratio first decreases and then increases. This is because both schemes do not use retransmissions when the message loss rate is below $20 \%$. Clearly, CPS and DPS do not exhibit the same behavior, since they use fixed parameters. Both the energy consumption (Figure 6b) and the 
latency (Figure 6c) increase with the message loss rate, since a higher number of messages has to be retransmitted due to channel errors. Moreover, the energy consumption of ADAPT is only slightly higher than OPS. The lower latency of OPS for a $10 \%$ message loss rate is also due to the specific values of the MAC parameters used in the corresponding conditions.

\section{B. Analysis in dynamic conditions}

In the following, we will investigate the performance of different schemes in the basic scenario of 20 nodes when the operating conditions change over time. Although we repeated the experiments several times, we only present the metrics of interest for a single representative simulation run (the results are found similar in other cases).

In the first set of experiments, we considered the scenario where the network workload changes over time, in terms of the number of messages sent by each node in a single BI. In detail, all nodes initially generated one message per BI. Then, at the $200^{\text {th }} \mathrm{BI}$, they increased the number of messages per BI to 5 . Next, at the $500^{\text {th }} \mathrm{BI}$, they generated 10 messages per BI. Finally, at the $800^{\text {th }}$ BI they reverted back to the original rate of one message per $\mathrm{BI}$.

We start by analyzing the dynamic network workload as a function of the BIs (Figure 7). Figure 7a shows the delivery ratio. It is easy to notice that DPS is not suitable for the considered scenario, since it attains a delivery ratio of at most $30 \%$. All other schemes provide a delivery ratio which is is always above the required reliability value, as intended. Also, here CPS achieves a $100 \%$ delivery ratio irrespective of the network workload. On the average, ADAPT has a delivery ratio closer to the required target of $80 \%$, especially when the workload is light (i.e., one message per BI). Figure $7 \mathrm{~b}$ shows the energy consumed per each message successfully received at the sink. Except for DPS, all other schemes have similar values of energy consumption. Again, here the energy consumption of ADAPT is only slightly higher than OPS, and (on the average) lower than CPS. Figure 7c shows the latency as a function of the BIs. Also here ADAPT obtains the lowest latency of around $100 \mathrm{~ms}$, which is much lower than that of both OPS and CPS. In addition, the latency is also quite stable, almost irrespective of the workload.

In the second set of experiments, we considered the scenario where the message loss rate changes over time. Specifically, the message loss rate was $0 \%$ between the initial and the $200^{\text {th }}$ $\mathrm{BI}$, then increased to $10 \%$ until the $500^{\text {th }} \mathrm{BI}$, and finally to $20 \%$ up to the $800^{\text {th }}$ BI. After the $800^{\text {th }}$ BI and until the end of the simulation, the message loss rate went back to the original value of $0 \%$.

The results with dynamic message loss rate as a function of the BIs are shown in Figure 8. In detail, Figure 8a shows the delivery ratio. In this case ADAPT is able to satisfy the required reliability constraint, and has a trend similar to OPS. Furthermore, the delivery ratio of CPS is very high, and lower than $100 \%$ only when the message loss rate is $20 \%$; on the other hand, the delivery ratio of DPS is very low, always below $20 \%$. Figure $8 \mathrm{~b}$ shows the energy consumed per each message successfully received at the sink. Clearly, OPS consumes the lowest energy during the whole experiment.
ADAPT has a very similar performance, with only slightly higher energy consumption than OPS. In any case, both OPS and ADAPT consume significantly lower energy than CPS, especially when the message loss rate is higher than $10 \%$. Finally, we consider the latency as shown in Figure 8c. Among the schemes achieving the highest delivery ratio, ADAPT has the lowest latency, which is always $50 \mathrm{~ms}$ below OPS.

\section{Convergence and impact of thresholds}

In the following we will evaluate the convergence and the sensitivity of ADAPT, in terms of whether (and how fast) it can effectively enforce the target delivery ratio, and how the performance is affected by the thresholds.

Let us first focus on the convergence. To this end, we performed a set of experiments under dynamic conditions, by following an approach similar to Section VII-B. Specifically, we assume that the message loss due to channel errors is negligible, and refer to the basic scenario where 20 nodes send one message per BI to the sink. We then suddenly change the network load from 1 to 5 messages per BI, and finally to 10 messages per BI. Every time the number of messages is increased, the MAC parameters are reset to their default values, in order to avoid any eventual bias introduced by prior tuning of the MAC parameters. The trend of the delivery ratio as a function of time is shown in Figure 9.

From the results we can see that the proposed scheme ADAPT always requires less than $12 \mathrm{BIs}$ to increase the delivery ratio above the desired level of reliability. In addition, once the target delivery ratio is reached, the actual delivery ratio almost always remains above the required reliability. This can also be derived from Observation 1. In particular, since an increase in the MAC parameter values leads to an increase of the reliability, and vice versa, the actual delivery ratio can be effectively kept in the region bounded by $d^{l o w}$ and $d^{h i g h}$. The only side effect is an oscillation which is indeed very low, and tends to decrease as the traffic increases, since the estimate of the delivery ratio is more accurate under loaded (or saturated) scenarios due to the larger number of messages sent. We also performed an additional set of experiments with different message loss rates (not shown here for the sake of brevity), which substantially confirm our findings. The main difference is that, when the message loss is high, retransmissions are triggered by the error control scheme, and thus the convergence to the target delivery ratio is slower but still below 15 BIs.

As for the sensitivity, we show the impact of the thresholds $\sigma$ and $\gamma$ on the performance in Tables II-a and II-b, respectively. We used the same basic scenario described above; as for the other parameters, we used the same values reported in Table I. In detail, we provide the average delivery ratio, the amount of times the delivery ratio is below the desired value (i.e., $d<d^{\text {des }}$ ) and the average energy consumed by a single node. Observe that, in both cases, increasing the thresholds leads to a higher average delivery ratio and, in turn, less "violations" of the desired delivery ratio. However, those violations are very limited, not exceeding $1.33 \%$ of 


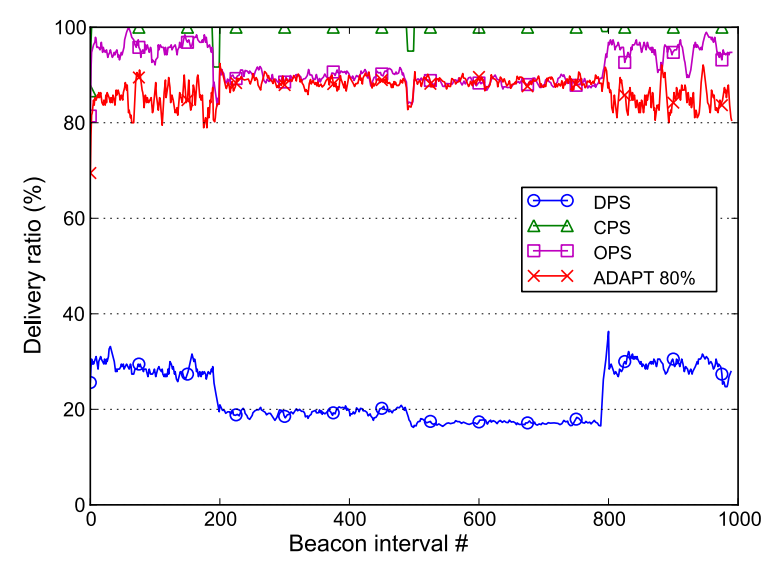

(a)

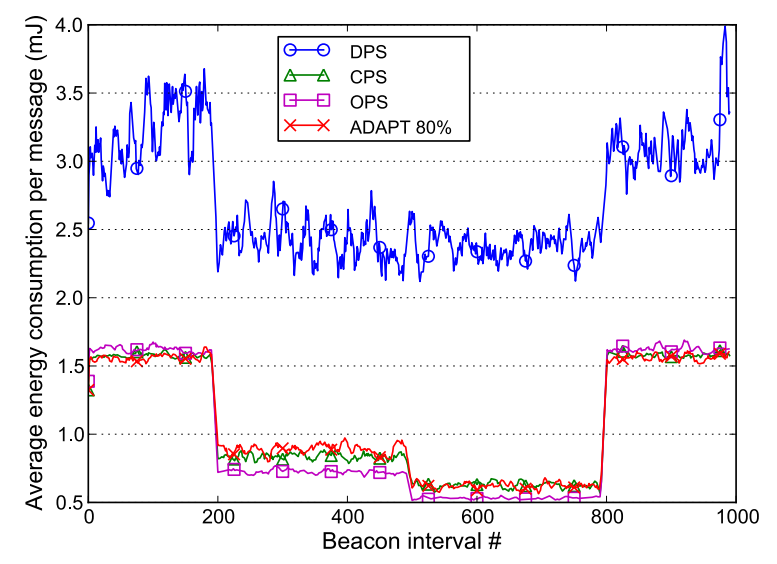

(b)

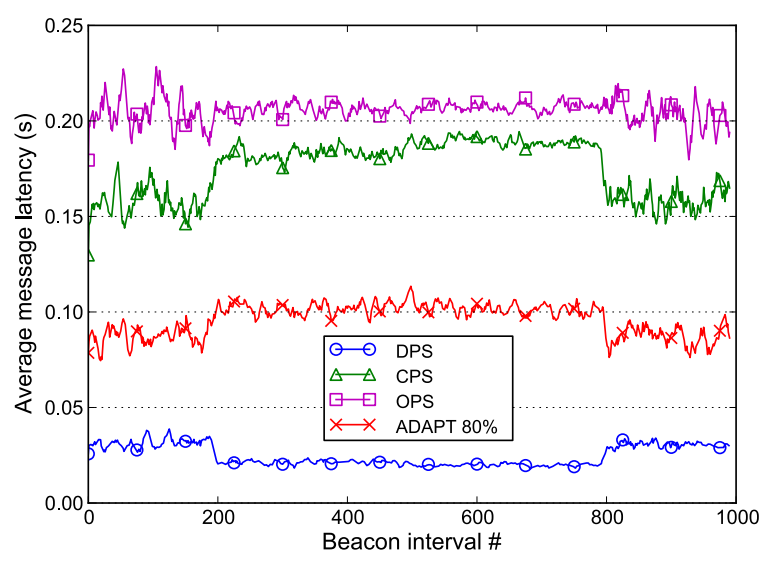

(c)

Fig. 7. Performance under dynamic number of messages per BI in the singlehop scenario: (a) delivery ratio, (b) energy consumption, and (c) latency.

the total measurements ${ }^{5}$. On the other hand, increasing the threshold results in a higher energy consumption per node.

${ }^{5}$ We have also performed additional simulations (not shown here for the sake of space) where we varied the message loss and the values of the other parameters as well. Even in the most critical scenarios, and for low values of both $\gamma$ and $\sigma$ (i.e., in the order of $1 \%$ ), the number of violations is always below $1.5 \%$.

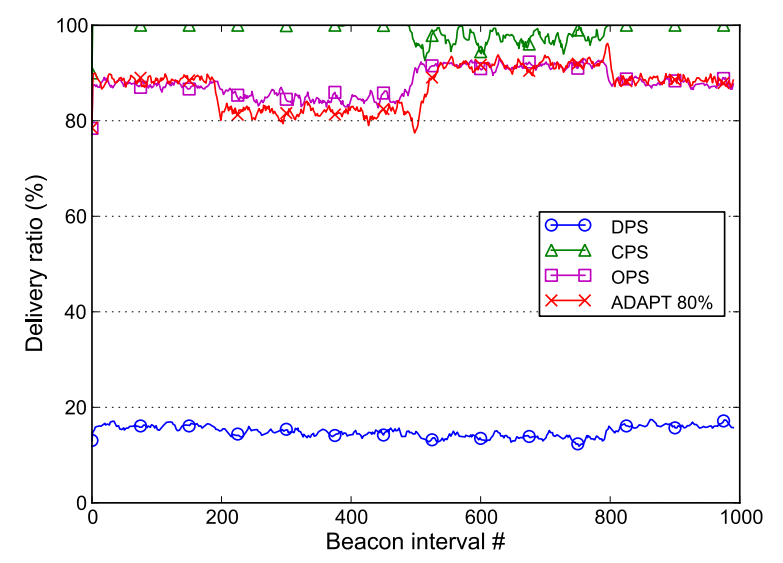

(a)

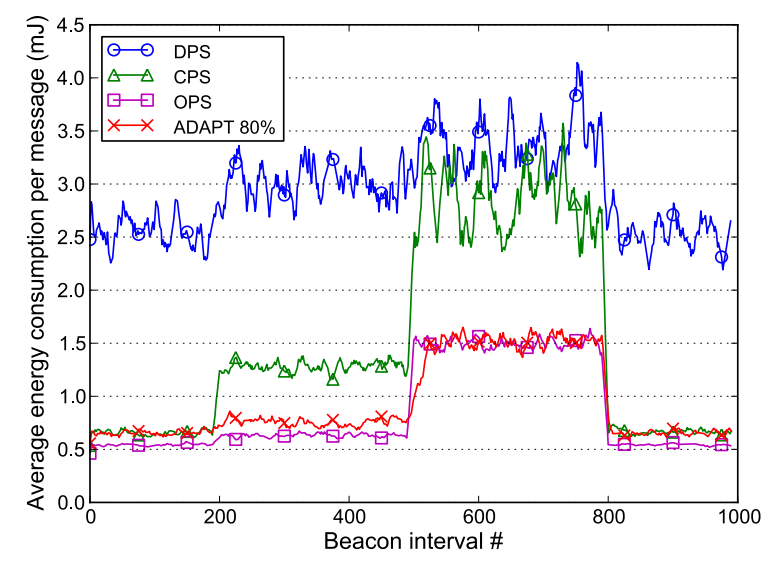

(b)

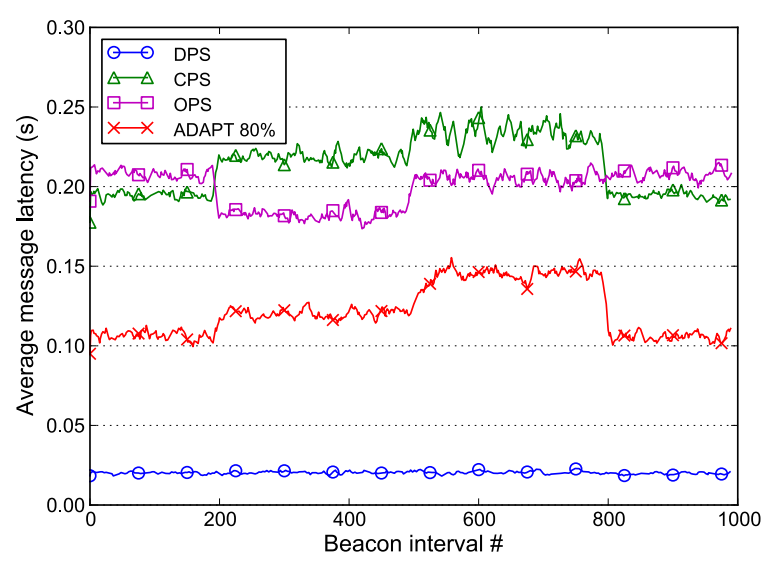

(c)

Fig. 8. Performance under dynamic message loss rate in the single-hop scenario: (a) delivery ratio, (b) energy consumption, and (c) latency.

This is also related to the trade-off between reliability and energy efficiency. By evaluating the joint impact of the two parameters, i.e., the ratio between the delivery ratio and the energy expenditure - the values of $\gamma$ and $\sigma$ equal to 3 usually achieve the best results in a variety of conditions, with a very low number of violations. Nevertheless, ADAPT 

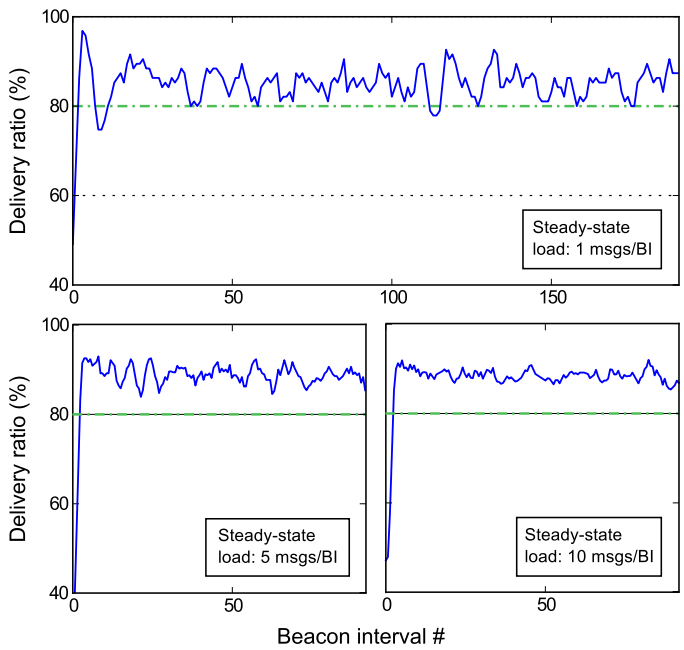

Fig. 9. Convergence as a function of the number of messages per BI.

is overall robust, almost irrespective of the actual values of the thresholds.

\section{MUlti-HOP SCENARIO}

The previous section dealt with the performance of different schemes in a single-hop scenario. In the following, we consider a multi-hop WSN based on the ZigBee specifications [3]. Specifically, we will consider a cluster-tree architecture, where the sensor nodes are organized into groups (clusters) according to their level in the routing tree. Each cluster is managed by a local coordinator (also called cluster-head), which exploits the beacon enabled mode of the IEEE 802.15.4 standard for energy conservation. As mentioned in Section III-B, the scheduling scheme for ZigBee networks operating in the beacon enabled mode requires that the active periods of (interfering) coordinators are not overlapped. As a consequence, each cluster is not affected by the contention originated by other clusters. Hence, the basic approach we have derived for single-hop (star) networks can be extended to multi-hop networks as well. Note that this approach would not have been possible if other scheduling schemes were used [7].

However, multi-hop networks also need additional mechanisms. In fact, the adaptive framework has considered local (link-based) parameters so far, while the application requirements are global (end-to-end). Hence, the (global) reliability requirements demand a mapping to the different regions of the network. In the following, we will first introduce a strategy for mapping the global reliability to local parameters, and then evaluate the performance of the adaptive framework in a multihop scenario.

\section{A. Global to local reliability mapping}

Let us consider a multi-hop path whose hop-by-hop link reliability is given by $d_{j}$ (referring to the $j$-th link), in terms of the delivery ratio. Since the messages are relayed hop-byhop by nodes from the source to the sink, the final (endto-end) reliability results from the individual link delivery ratios. Assuming that the link reliability of individual paths
TABLE II

IMPACT OF $\sigma$ AND $\gamma$ ON THE PERFORMANCE

(a)

\begin{tabular}{|c|l|l|l|}
\hline $\begin{array}{c}\sigma \\
(\%)\end{array}$ & $\begin{array}{l}\text { Delivery } \\
\text { ratio (\%) }\end{array}$ & $\begin{array}{l}d<d^{\text {des }} \\
(\boldsymbol{\%})\end{array}$ & $\begin{array}{l}\text { Energy } \\
(\mathbf{J} / \mathbf{n o d e})\end{array}$ \\
\hline \hline 1 & 83.17 & 1.33 & 1.443 \\
\hline 2 & 83.30 & 1.27 & 1.444 \\
\hline 3 & 83.51 & 1.28 & 1.446 \\
\hline 4 & 84.29 & 1.16 & 1.461 \\
\hline 5 & 84.56 & 1.11 & 1.468 \\
\hline 6 & 84.76 & 1.12 & 1.469 \\
\hline
\end{tabular}

(b)

\begin{tabular}{|c|l|l|l|}
\hline $\begin{array}{c}\gamma \\
(\%)\end{array}$ & $\begin{array}{l}\text { Delivery } \\
\text { ratio }(\boldsymbol{\%})\end{array}$ & $\begin{array}{l}d<d^{\text {des }} \\
(\boldsymbol{\%})\end{array}$ & $\begin{array}{l}\text { Energy } \\
(\mathbf{J} / \text { node })\end{array}$ \\
\hline \hline 1 & 83.25 & 1.33 & 1.443 \\
\hline 2 & 83.34 & 1.23 & 1.443 \\
\hline 3 & 83.51 & 1.28 & 1.446 \\
\hline 4 & 84.16 & 1.18 & 1.458 \\
\hline 5 & 84.45 & 1.13 & 1.463 \\
\hline 6 & 84.43 & 1.09 & 1.464 \\
\hline
\end{tabular}

are independent and have the same value, i.e., $d_{j}=\bar{d}$ for any $j$, the end-to-end reliability is given by

$$
d^{\text {path }}=\prod_{k=1}^{n} d_{k}=(\bar{d})^{n}
$$

where $n$ is the number of hops between the source and the destination. As a result, the nodes have an end-to-end reliability which decreases when the number of hops (hence the distance from the sink) increases. Consequently, the reliability requirement cannot be satisfied just by enforcing the same value of the target delivery ratio at all hops, otherwise nodes far away from the sink would be penalized.

In order to solve these issues, we have defined a reliability mapping scheme which exploits information from the routing layer to set the target link delivery ratio at each cluster. Specifically, we set the link-level (required) reliability of a message originated by a node which is $j$ hops away from the sink to

$$
d_{j}^{d e s}=\sqrt[j]{d^{d e s}}
$$

As a consequence, individual messages are assigned a specific target reliability which depends on the distance of the originating node from the sink. Note that this is just one of the possible choices which are supported by our adaptive framework.

We apply the above mapping to both OPS and ADAPT. Due to limitations of the analytical model, the target delivery ratio is assigned for OPS according to the cluster level, i.e., the hop distance of all nodes in the cluster from the sink. Instead, the target delivery ratio is assigned for ADAPT on a message-by-message basis, according to the actual hop distance of the source node to the sink. Since messages are relayed through the multi-hop path, the related information is included in the message itself through a header field, otherwise it would be impossible for the relaying node to enforce the proper value of the delivery ratio. In detail, each node running ADAPT operates as follows. It first obtains the required endto-end delivery ratio $d^{d e s}$ from the application. Then, for each message generated by itself, it applies the adaptive policy described above with the help of the target delivery ratio $d_{o}^{\text {des }}$ 


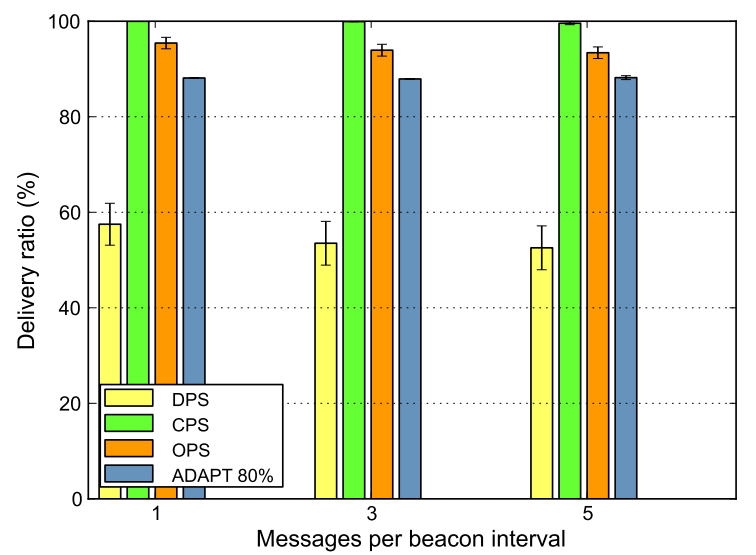

(a)

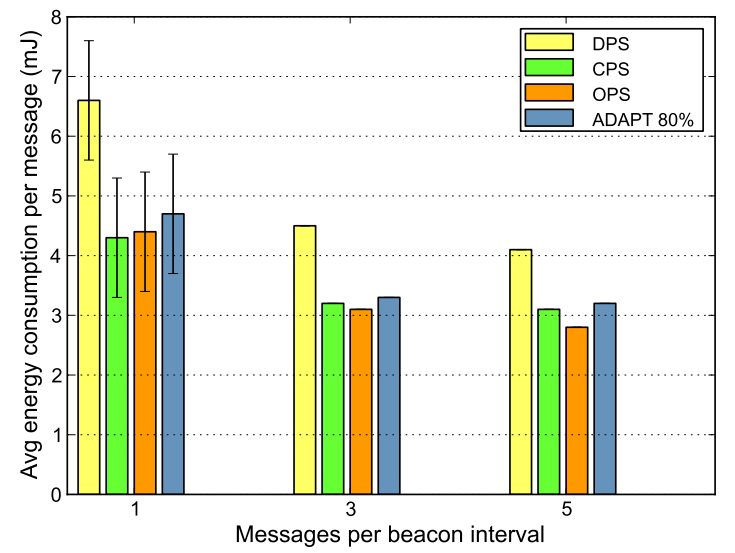

(b)

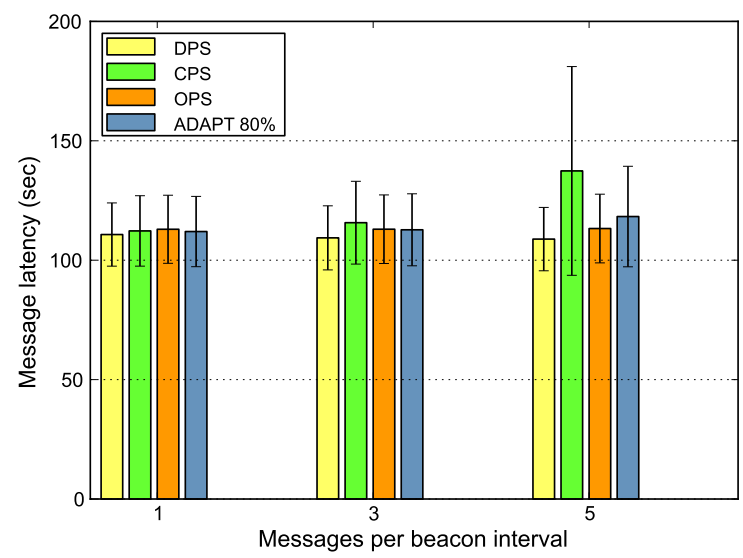

(c)

Fig. 10. Performance as a function of the number of messages per BI in the multi-hop scenario: (a) delivery ratio, (b) energy consumption, and (c) latency.

as defined by Equation (3), where $o$ is its own hop distance from the sink. Instead, for each relayed message, the node first inspects the header and obtains the hop distance $h$ of the sender node from the sink, and then it applies the adaptive policy by using the target delivery ratio $d_{h}^{\text {des }}$ according to Equation (3).

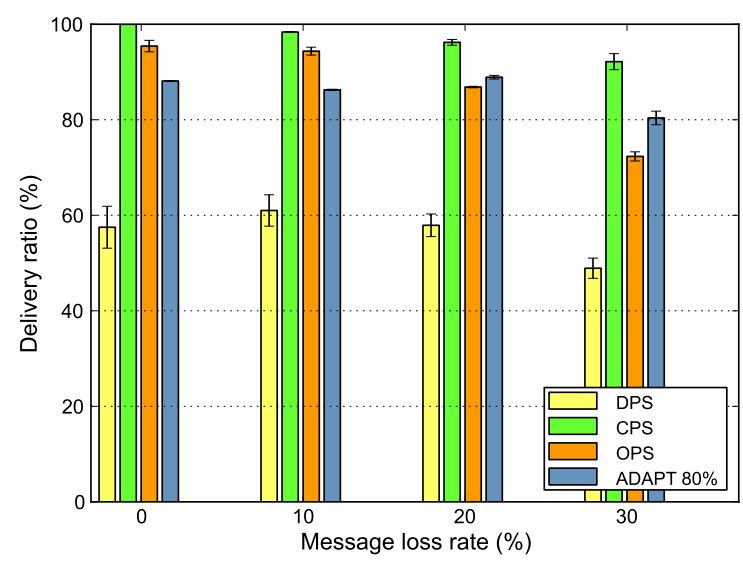

(a)

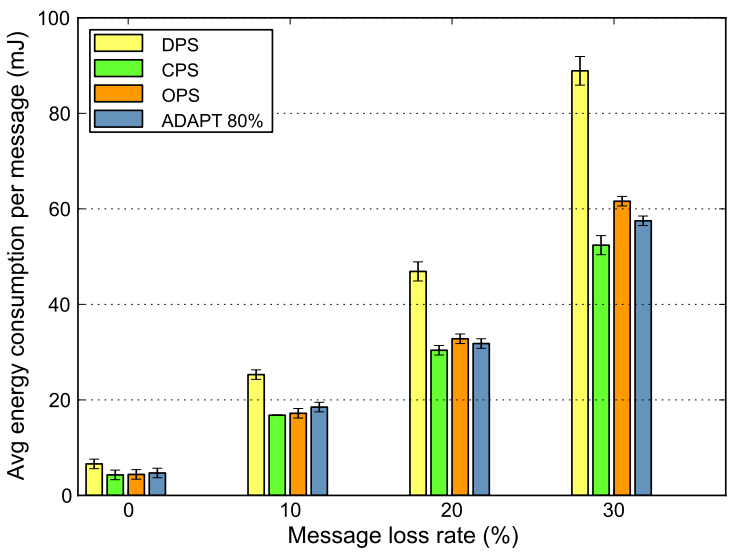

(b)

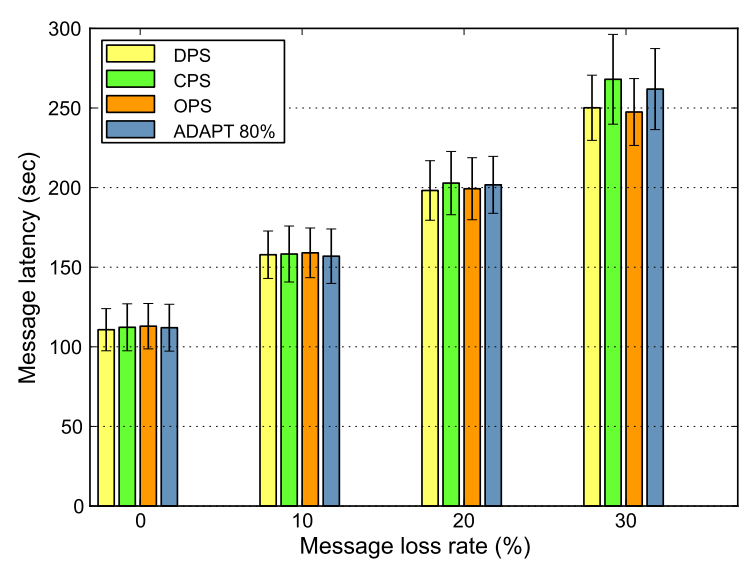

(c)

Fig. 11. Performance as a function of the message loss rate in the multi-hop scenario: (a) delivery ratio, (b) energy consumption, and (c) latency.

\section{B. Simulation setup}

We considered a WSN where 100 sensor nodes were randomly deployed in a $100 \mathrm{~m} \times 100 \mathrm{~m}$ area. We implemented a simple tree construction algorithm based on the minimum hop count to organize the sensor nodes in a logical tree (the routing protocol produces a tree with 7 levels on the average 
for the considered scenario). The sink is the PAN coordinator, and the non-leaf nodes are the cluster coordinators. All other devices are ordinary nodes associated with their own cluster coordinator. Due to the increased amount of traffic resulting from the large number of nodes and the multi-hop message forwarding process, we increased the active period, namely the superframe duration SD, to $15.7 \mathrm{~s}$. All other parameters are as specified in Section VI, and we also consider here a target delivery ratio of $80 \%$.

As for the multi-hop duty-cycle coordination, we considered a ZigBee-compliant superframe scheduling scheme [3]. Specifically, we scheduled the active portions of the superframe in a TDMA-like fashion, so that only a single parent and its children are simultaneously active in the network. In addition, the superframes are arranged such that all messages are collected by a single node from its children before it starts communicating with its parent [7].

\section{Experimental results}

In the first set of experiments we evaluated the impact of the number of messages per BI on the performance (see Figure 10). As for the delivery ratio, shown in Figure 10a, we observe that ADAPT can effectively satisfy the required reliability. Furthermore, the number of messages for BI does not significantly affect the delivery ratio, substantially confirming the trend of Figure 5a in multi-hop scenarios. A similar trend appears in Figure 10b, which shows the energy consumption per each message correctly received by the sink. While the energy expenditure is higher when the nodes send one message per BI, it is almost the same when the workload increases. In all cases, ADAPT consumes energy very close to the optimal value (OPS), even though CPS performs better in this scenario. However, in a multi-hop scenario, it is important to consider the total energy consumed by the one-hop neighbors of the sink, which actually determine the network lifetime, rather than the energy per delivered messages. We anticipate here that the total energy consumption of CPS is higher than ADAPT (cfr. Figures 12b and Figure 12c). As for the latency, depicted in Figure 10c, we can see that all schemes have almost the same performance, irrespective of the workload. This happens because in the multi-hop scenario, the major contribution to latency is given by the sleep/wakeup scheduling (or dutycycling) protocol, hence by the duration of the active periods of the superframes. In fact, before being relayed to the nodes in the tree toward the sink, the messages are queued while waiting for the next hop to wake up [4].

In the second set of experiments, we considered the performance as a function of the message loss rate (see Figure 11). Specifically, the delivery ratio is plotted in Figure 11a. In this case, ADAPT obtains a delivery ratio above the required $80 \%$ reliability, and substantially independent of the message loss rate. Both OPS and CPS are more sensitive to the message loss rate. Specifically, OPS is negatively affected by a high message loss, resulting in a low delivery ratio when the message loss rate is equal to $30 \%$. We now consider the energy consumption per received message, shown in Figure 11b. Besides the fact that the energy consumption increases almost linearly with the message loss rate, we can see that

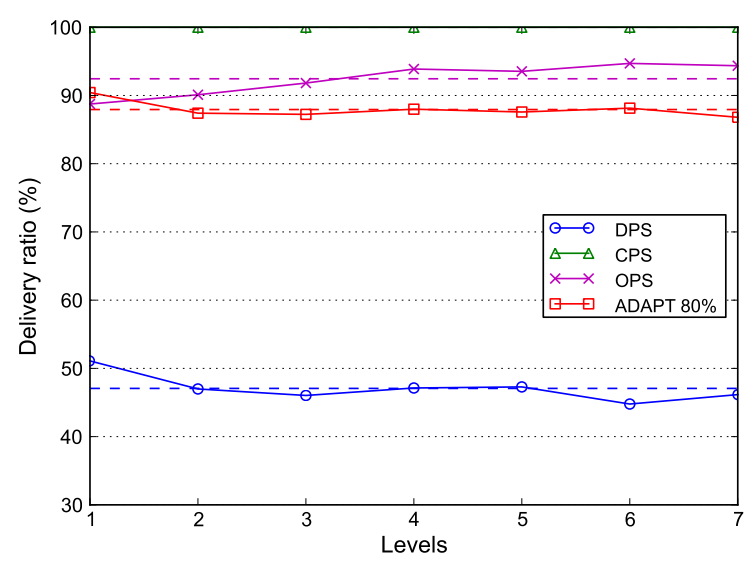

(a)

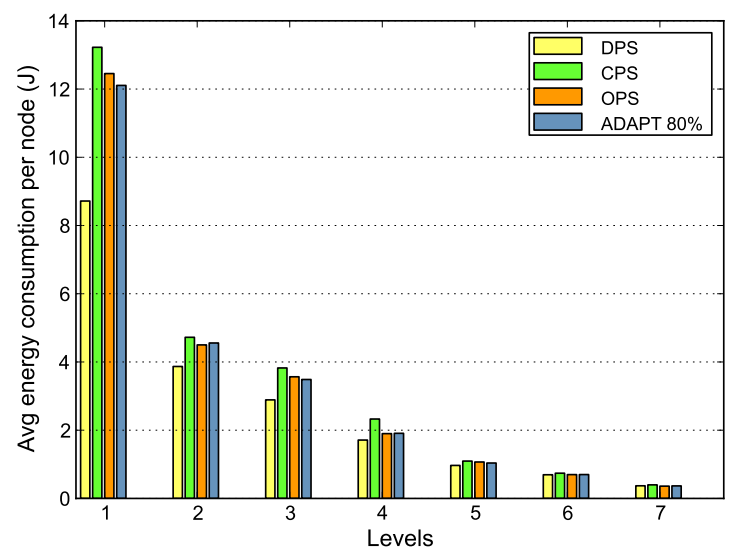

(b)

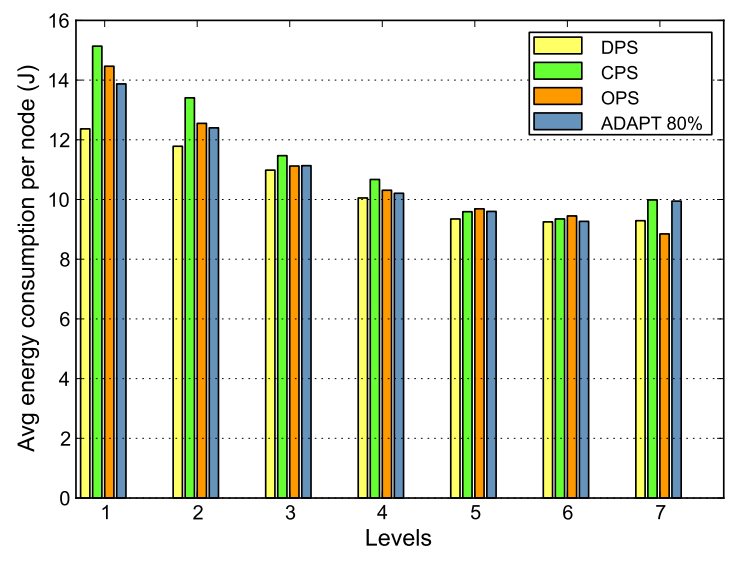

(c)

Fig. 12. Performance as a function of the hop distance: (a) delivery ratio, (b) energy consumption with $0 \%$ message loss rate, and (c) $20 \%$ message loss rate.

OPS actually consumes more energy than ADAPT when the message loss rate is equal to $20 \%$. This is due to the limitations of the model, which lacks flexibility since it can only operate at the cluster level. As a consequence, the reliability mapping scheme cannot consider delivery ratios different from message to message (i.e., for messages originated by other clusters). 
Therefore, all messages either generated or relayed by a node at a certain distance from the sink are assigned the same level of target reliability, which is not enough to meet the end-toend requirements. Finally, Figure 11c shows that the latency is independent of the scheme used, and increases linearly with the message loss rate, different from Figure 10c. This happens because a higher message loss rate triggers the retransmission mechanism, which increases the channel access time. As a result, messages may not be forwarded to the next hop during a single active period. In this case, the nodes have to wait for the next active period before transmitting those messages, which results in an additional delay corresponding to the BI [7].

We finally focus on the fairness of the different schemes, in terms of how the delivery ratio and the energy consumption are distributed among the nodes with different hop distances from the sink (see Figure 12). Let us first discuss the delivery ratio as a function of the hop count, as illustrated in Figure 12a. We can see that, different from both OPS and DPS, the proposed scheme ADAPT has a very low variation of the delivery ratio around the average value (depicted as a dashed line in the figure), even though CPS obtains a $100 \%$ delivery ratio irrespective of the hop count. Similar results, not shown here due to space limitations, have been obtained with different values of message loss rate and number of messages per BI. Figure $12 \mathrm{~b}$ shows the energy consumed by nodes at different levels in the routing tree. Clearly, the one-hop neighbors of the sink are the most loaded nodes in the network. Interestingly, ADAPT has the lowest energy consumption for the one-hop neighbors of the sink, while the energy expenditure of OPS is slightly higher, and that of CPS is even larger. As a consequence, since the one-hop neighbors of the sink determine the network lifetime, ADAPT can increase the longevity of the network. The same trend appears in the lower levels of the tree, where the distance between the different schemes is clearly reduced. ADAPT consumes the lowest energy per node when the message loss rate is $20 \%$, according to Figure $12 \mathrm{c}$, even though the energy consumption for the one-hop neighbors of the sink is comparable to the one at the lower levels in the tree. This happens because the channel access time increases, as already explained above. Hence the total activity of the nodes increases too, basically independent of the level of the tree.

\section{CONCLUSIONS}

In this paper we have proposed an adaptive framework for reliable and energy-efficient data collection in wireless sensor networks based on the IEEE 802.15.4/ZigBee standards. Our framework exploits a cross-layer approach, and guarantees that the reliability value specified by the application (in terms of a target delivery ratio) is met with a low energy consumption. By collecting information available at both the routing and the MAC layers, our adaptive framework autonomously tunes the parameters of the IEEE 802.15.4 MAC protocol without requiring any modification to the standard. The proposed framework supports multi-hop wireless sensor networks based on the ZigBee specifications, and is flexible enough to implement diverse adaptation policies. In particular, we have designed a fully distributed and low-overhead ADaptive
Access Parameters Tuning (ADAPT) algorithm based on an analytical performance evaluation of the IEEE 802.15.4 MAC protocol.

Simulation results demonstrated that ADAPT can effectively enforce the required delivery ratio under a wide range of operating conditions, for both single-hop and multi-hop scenarios. In addition, due to its ability to automatically adapt to the network demands, ADAPT results in a near-optimal energy consumption and in a very low latency. Finally, its adaptation policy is extremely lightweight, thus well suited for implementation on real sensor nodes. We are currently implementing ADAPT on a testbed of Mote sensors. Preliminary results confirmed those obtained from simulations.

\section{ACKNOWLEDGEMENT}

We are extremely grateful to the anonymous reviewers for their constructive suggestions, which helped us to significantly improve the quality of the manuscript.

\section{REFERENCES}

[1] Embedded WiSeNTs Consortium, "Embedded WiSeNts Research Roadmap (Deliverable 3.3)," http://www.embedded-wisents.org/ dissemination/roadmap.html, 2006.

[2] "IEEE 802.15.4, Part 15.4: Wireless Medium Access Control (MAC) and Physical Layer (PHY) Specifications for Low-Rate Wireless Personal Area Networks (LR-WPANs)," September 2006, revision of IEEE Std 802.15.4-2003.

[3] “The ZigBee Specification version 2.0," December 2006.

[4] G. Anastasi, M. Conti, M. Di Francesco, and A. Passarella, "Energy conservation in wireless sensor networks: A survey," Ad Hoc Networks, vol. 7, no. 3, pp. 537-568, May 2009.

[5] A. Willig, "Recent and emerging topics in wireless industrial communications: A selection," IEEE Trans. Ind. Informat., vol. 4, no. 2, pp. 102-124, May 2008.

[6] G. Anastasi, M. Conti, and M. Di Francesco, "A comprehensive analysis of the MAC unreliability problem in IEEE 802.15.4 wireless sensor networks," IEEE Trans. Ind. Informat., vol. 7, no. 1, pp. 52-65, February 2011.

[7] G. Anastasi, M. Conti, M. Di Francesco, and V. Neri, "Reliability and energy efficiency in multi-hop IEEE 802.15.4/ZigBee wireless sensor networks," in Proc. $15^{\text {th }}$ IEEE Symposium on Computers and Communications (ISCC 2010), Rimini, Italy, 22-25 June 2010.

[8] M. Di Francesco, G. Anastasi, M. Conti, S. K. Das, and V. Neri, "An adaptive algorithm for dynamic tuning of MAC parameters in IEEE 802.15.4/ZigBee sensor networks," in Proc. of the $6^{\text {th }}$ IEEE International Workshop on Sensor Networks and Systems for Pervasive Computing (PerSeNS 2010), 29 March 2010.

[9] T. Melodia, M. C. Vuran, and D. Pompili, "The state of the art in crosslayer design for wireless sensor networks," in Proc. EuroNGI Workshops on Wireless and Mobility. Springer Lecture Notes in Computer Science 3883 , July 2005.

[10] J. Misic, S. Shafi, and V. Misic, "Cross-layer activity management in an 802-15.4 sensor network," IEEE Commun. Mag., vol. 44, no. 1, pp. 131-136, January 2006.

[11] W. Su and T. Lim, "Cross-layer design and optimization for wireless sensor networks," in Proc. of the $7^{\text {th }}$ ACIS International Conference on Software Engineering, Artificial Intelligence, Networking, and Parallel/Distributed Computing (SNPD 2006), June 2006, pp. 278-284.

[12] K. Yedavalli and B. Krishnamachari, "Enhancement of the IEEE 802.15.4 MAC protocol for scalable data collection in dense sensor networks," in Proc. of the $6^{\text {th }}$ International Symposium on Modeling and Optimization in Mobile, Ad Hoc, and Wireless Networks and Workshops (WiOPT 2008), April 2008, pp. 152-161.

[13] C. K. Singh, A. Kumar, and P. M. Ameer, "Performance evaluation of an IEEE 802.15.4 sensor network with a star topology," Wireless Networks, vol. 14, no. 4, pp. 543-568, 2008.

[14] S. Pollin, M. Ergen, S. Ergen, B. Bougard, L. Der Perre, I. Moerman, A. Bahai, P. Varaiya, and F. Catthoor, "Performance analysis of slotted carrier sense IEEE 802.15.4 medium access layer," IEEE Trans. Wireless Commun., vol. 7, no. 9, pp. 3359-3371, September 2008. 
[15] P. Park, C. Fischione, and K. Johansson, "Adaptive IEEE 802.15.4 protocol for energy efficient, reliable and timely communications," in Proc. $9^{\text {th }}$ International Conference onInformation Processing in Sensor Networks (IPSN 2010), April 12-16 2010, pp. 327-338.

[16] P. Park, P. Di Marco, P. Soldati, C. Fischione, and K. Johansson, "A generalized markov chain model for effective analysis of slotted IEEE 802.15.4," in Proc. $6^{\text {th }}$ IEEE International Conference on Mobile Adhoc and Sensor Systems (MASS '09), October 2009, pp. $130-139$.

[17] G. Anastasi, M. Conti, and M. Di Francesco, "Extending the lifetime of wireless sensor networks through adaptive sleep," IEEE Trans. Ind. Informat., vol. 5, no. 3, pp. 351-365, August 2009.

[18] I. Ramachandran, A. K. Das, and S. Roy, "Analysis of the contention access period of IEEE 802.15.4 MAC," ACM Trans. Sensor Networks, vol. 3, no. 1, 2007.

[19] M. Conti, G. Maselli, G. Turi, and S. Giordano, "Cross-layering in mobile ad hoc network design," Computer, vol. 37, pp. 48-51, February 2004.

[20] V. Srivastava and M. Motani, "Cross-layer design: a survey and the road ahead," IEEE Commun. Mag., vol. 43, no. 12, pp. 112-119, December 2005.

[21] J. F. Kurose and K. W. Ross, Computer Networking - A Top-Down Approach Featuring the Internet, 5th ed. Addison-Wesley Professional, 2009.

[22] G. Anastasi, E. Borgia, M. Conti, E. Gregori, and A. Passarella, "Understanding the real behavior of Mote and 802.11 ad hoc networks: an experimental approach," Pervasive and Mobile Computing, vol. 1, no. 2, pp. 237-256, 2005 .

[23] "Network Simulator version 2," http://www.isi.edu/nsnam/ns/.

[24] A. Willig, M. Kubisch, C. Hoene, and A. Wolisz, "Measurements of a wireless link in an industrial environment using an IEEE 802.11compliant physical layer," IEEE Trans. Ind. Electron., vol. 49, no. 6, pp. 1265-1282, dec 2002.

[25] Texas Instruments, "CC2420 2.4 GHz IEEE 802.15.4 / ZigBee-Ready RF Transceiver," http://focus.ti.com/docs/prod/folders/print/cc2420. html.

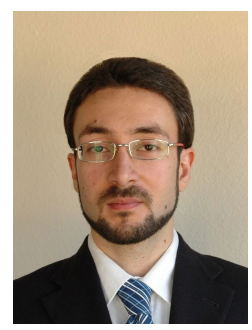

Mario Di Francesco is a Research Associate with the Department of Computer Science and Engineering, University of Texas at Arlington. He received his Ph.D. degree from the Department of Information Engineering at the University of Pisa, Italy, in 2009. He was also a Research Assistant with the Real-Time Systems (ReTIS) Laboratory at the Scuola Superiore S. Anna in 2009. His current research interests include pervasive computing and wireless sensor networks. Dr. Di Francesco was a TPC member of PerCom 2010, and has been a TPC member of IEEE PerSeNS since 2009. He also served as publication Co-Chair of IEEE WoWMoM 2006.

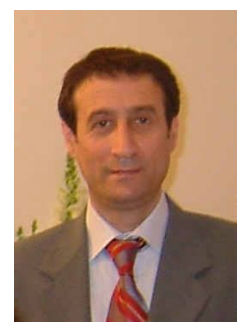

Giuseppe Anastasi is an Associate Professor of Computer Engineering at the Department of Information Engineering of the University of Pisa, Italy. He received the MS degree in Electronics Engineering, and the $\mathrm{PhD}$ degree in Computer Engineering, both from the University of Pisa, in 1990 and 1995, respectively. His research interests include pervasive computing, wireless sensor and actuator networks, and sustainable computing. He is the founding cochair of the Pervasive Computing \& Networking Laboratory (PerLab) in Pisa, and has contributed to many research programs funded by both national and international institutions. $\mathrm{He}$ is a co-editor of the book "Advanced Lectures in Networking" (LNCS 2497, Springer, 2002), and has published about 100 research papers in the area of computer networking. He is an Area Editor of Sustainable Computing (SUSCOM) and Pervasive and Mobile Computing (PMC), and is currently serving as Program Chair of the First IEEE Workshop on Sustainable Internet and Internet for Sustainability (SustaInet 2011). He has served as Area Editor of Computer Communications (ComCom), General Co-chair of IEEE WoWMoM 2005, Program Chair of IEEE PerCom 2010 and IEEE WoWMoM 2008, and Vice Program Chair of IEEE MASS 2007. He has launched the IEEE Workshop on Sensor Networks and Systems for Pervasive Computing (PerSeNS), co-located with IEEE PerCom. He has been a member of the IEEE Computer Society since 1994.

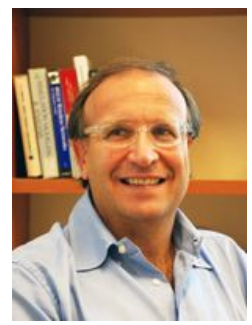

Marco Conti is a Research Director at the Institute of Informatics and Telematics (IIT), an Institute of the Italian National Research Council (CNR). He published in journals and conference proceedings more than 250 research papers related to design, modeling, and performance evaluation of computernetwork architectures and protocols. He coauthored the book "Metropolitan Area Networks" (1997) and is co-editor of the books "Mobile Ad Hoc Networking" (2004) and "Mobile Ad Hoc Networks: From Theory to Reality (2007). He is the founding cochair of the Pervasive Computing \& Networking Laboratory (PerLab) in Pisa. He is the Chair of the IFIP WG 6.3 "Performance of Communication Systems.". Dr. Conti is the Editor-in-Chief of the Computer Communications Journal and Associate Editor-in-Chief of the Pervasive and Mobile Computing Journal. He is on the Editorial Board of Ad Hoc Networks, Journal of Communications Systems, and Wireless Ad Hoc and Sensor Networks. He served as General Chair of ACM REALMAN 2006 and IEEE MASS 2007, and as General Co-Chair of IEEE WoWMoM 2006, ACM MobiOpp 2007, and IEEE PerCom 2010. He served as TPC Chair of IEEE PerCom 2006, and of the IFIP-TC6 conferences Networking 2002 and PWC 2003, and as TPC Co-Chair of ACM WoWMoM 2002, WiOpt 2004, IEEE WoWMoM 2005, ACM MobiHoc 2006, and ACM MobiOpp 2010. He is the founder of the ACM REALMAN and ACM MobiOpp workshop series and and the co-founder of the IEEE AOC workshop series. He is currently serving as General Co-chair of the First IEEE Workshop on Sustainable Internet and Internet for Sustainability (SustaInet 2011) 


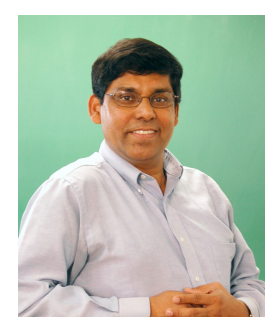

Sajal K. Das is a University Distinguished Scholar Professor of Computer Science and Engineering and the Founding Director of the Center for Research in Wireless Mobility and Networking (CReWMaN) at the University of Texas at Arlington (UTA). He is currently a Program Director at the US National Science Foundation (NSF) in the Division of Computer Networks and Systems. His current research interests include wireless and sensor networks, mobile and pervasive computing, smart environments and smart heath care, pervasive security, biological networking, applied graph theory and game theory. He has published over 400 papers and over 35 invited book chapters in these areas. He holds five US patents in wireless networks and mobile Internet, and coauthored the books "Smart Environments: Technology, Protocols, and Applications" (Wiley, 2005), "Mobile Agents in Distributed Computing and Networking" (Wiley, 2011), and Handbook on Cyber-Physical Infrastructure Security". $\mathrm{He}$ is a recipient of the IEEE Computer Society Technical Achievement Award (2009) for pioneering contributions to sensor networks. He serves as the Founding Editor-in-Chief of Elsevier's Pervasive and Mobile Computing (PMC) journal, and also as an Associate Editor of IEEE Transactions on
Mobile Computing, ACM/Springer Wireless Networks, Journal of Parallel and Distributed Computing, and Journal of Peer-to-Peer Networking. He is the founder of IEEE WoWMoM symposium and co-founder of IEEE PerCom conference. He is a senior member of the IEEE.

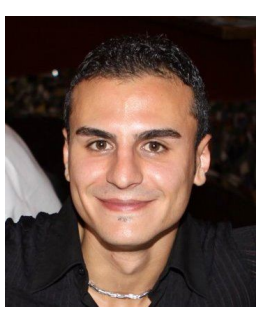

Vincenzo Neri received his B.S. and M.S. degrees in computer engineering from the University of Pisa, Italy, in 2006 and 2009, respectively. He was a Research Fellow with the Department of Information Engineering at the University of Pisa, Italy, in 2010. $\mathrm{He}$ is currently a System Analyst at the Avionics Laboratory of the company Ingegneria dei Sistemi (IDS). His research interests include performance evaluation of protocols for wireless sensor networks and simulation of complex systems. 